\title{
Sprachliche Erforschung der indischen Medizin
}

\author{
Von Rembert Watermann, Neuß-Reuschenberg*
}

\section{Skizze der indischen Sprachen}

Um sein Gegenüber richtig zu verstehen, bedarf es der Kenntnis seiner Sprache. Eine fremde Sprache zu erlernen, ist nicht leicht - eine fremde Grammatik genau zu beherrschen sogar sehr schwer. Im folgenden soll skizziert werden, wie lange es in der Geschichte gedauert hat, bis Europäer das Studium der indischen medizinischen Literatur sprachlich in Angriff nahmen und wie man sich vorher beholfen hat.

Mit den Portugiesen kamen schon früh Ärzte, Chirurgen und Barbiere nach Indien. Sie gewannen schnell Kontakt mit der Bevölkerung; von dem ersten europäischen Arzt ist sogar bekannt, daß er eine Inderin heiratete (1).

Die Europäer fanden Indien in viele Königreiche und Fürstentümer aufgeteilt. Die einzelnen Völker und Volksgruppen (Kasten ${ }^{1}$ ) sprachen zum Teil voneinander sehr verschiedene Sprachen, die oftmals ihre eigenen Schriftzeichen hatten. Wieder andere Völker besaßen keine eigene Sprache, oder aber sie hatten eine eigene Sprache, konnten diese aber nicht schreiben. Alte Reiseberichte zeugen davon, wie es nicht leicht war, sich in dem sprachlichen und völkischen Gewirr zurechtzufinden.

Aus der Vielheit der Reiche, Völker und Volksgruppen (Kasten) hoben sich dem Neuankömmling aber schnell die Brahmanen heraus, ein Volk, das sich zu Beginn der geschichtlichen Zeit von Nordwesten her nach und nach über fast alle andern Völker des indischen Subkontinents ausgebreitet haben soll und das schließlich die geistige Führung Indiens übernahm. Diese Überschichtung geschah nicht nur militärisch, sondern auch in ziviler Weise, indem die Fürsten, besonders im Süden Indiens, die Brahmanen als Ratgeber, Gelehrte und Schreibgewandte in ihre Reiche beriefen. Diese gelehrten Brahmanen brachten ihre eigene Literatur mit und erweiterten sie ständig. Was ihnen besonders wertvoll schien, wurde in der Kunstsprache Sanskrit aufgeschrieben. Sanskrit-Literatur gibt es von den Gegenden nördlich des Ganges bis hinab nach Ceylon. Man sagt, Sanskrit sei nicht nur eine für die Literatur besonders zurechtgemachte Sprache gewesen, sei

* Meinem Lehrer in der Ethnologie, Herrn Professor H. Trimborn, Bonn, in Verehrung gewidmet.

${ }^{1}$ Kaste kommt von Casta, f., portugiesisch $=$ Art, Geschlecht, Volksgruppe. 
nicht nur in der Dichtung und Wissenschaft verwendet, sondern auch tatsächlich gesprochen worden. Hochkastige Leute, so Könige, Fürsten, Minister, Brahmanen und Generäle hätten sich ihrer bedient. Es sei nur eine Sprache der Männer gewesen. Ihre Frauen, die Kinder und die verarmten oder niedrigstehenden Männer der gleichen brahmanischen Kaste hätten dagegen Prakrit gesprochen. Dieses leite sich vom Sanskrit ab, sei weniger gekünstelt und zweckmäßiger für den Umgang. Es gibt verschiedene Prakrit-Dialekte, auch Sprachen genannt. Nach anderer Meinung war Sanskrit die Sprache allein der brahmanischen Gelehrten; alle andern Männer und Frauen, die Könige, Minister, Generäle usw. hätten einen der PrakritDialekte gesprochen, selbst wenn sie brahmanischer Herkunft waren. Heutzutage kann keineswegs jeder Brahmane Sanskrit lesen und übersetzen, geschweige denn sprechen (2). Und schließlich nochmals die Frage, ob Sanskrit überhaupt jemals gesprochen worden ist: hierüber gibt es die Auffassung, daß es lediglich ein zum Schreiben von Dichtung und wissenschaftlicher Literatur verfeinertes, zurechtgemachtes Prakrit sei.

Prakrit gilt also auf jeden Fall als vulgärer als das Sanskrit. Es gibt verschiedene Sprachen, die Prakrit sind. Ihnen allen ist gemeinsam, daß sie mit dem Sanskrit verwandt sind, sich zum Teil sogar direkt von ihm ableiten sollen. Eine Prakrit-Sprache ist beispielsweise das Maharastri oder Mahratti, das von den östlich von Bombay lebenden Mahratten gesprochen wird. Die Prakrit-Sprachen, oft arische Sprachen genannt, dominieren im Norden und in Mittelindien. Im Hinblick auf die alte Literatur ist unter den Prakrits das Pali am bedeutendsten. Es gelangte durch die Buddhisten und Jainas nach dem Süden bis nach Ceylon, wo es den Anhängern dieser Religionen noch heute als heilige Sprache gilt.

Im Raume südlich von Goa, Haiderabad und von Orissa bis nach Ceylon hin dominieren die Drawida- oder nichtarischen Sprachen. Die Literatursprachen unter ihnen haben Schriftzeichen, die sich von denen der arischen Sprachen unterscheiden. Zu dieser südlichen Sprachgruppe gehören unter anderm das Malajalam, das Tamil und das Telugu (vgl. Sprachenskizze). Bei den Drawida-Völkern gibt es, wie oben bereits angedeutet, Brahmanen, deren Pandits die Sanskrit-Literatur betreiben. Es ist bemerkenswert, daß alle diese südlichen Pandits das Sanskrit keineswegs in einer einheitlichen Schrift schreiben. Sie wenden, wie es HeInz Bechert 1959 sehr eindrucksvoll geschildert hat (3), in jedem Teilbereich des südindischen Kulturraumes die regional übliche Schrift an. Derselbe Sanskrit-Text wird also im Tamil-Gebiet mit andern Schriftzeichen geschrieben als im Telugu-Gebiet. 
Außer den europäischen Sprachen spielt in Indien noch eine andere, ursprünglich nichtindische Sprache eine große Rolle, das Persische. Es wird heute noch mit arabischen Schriftzeichen geschrieben. Sein ehedem mächtiger Einfluß hat sich auf die arischen Sprachen Nord- und Mittelindiens, so auf das Hindi ausgewirkt. Viele Wörter sind aus dem Persischen und indirekt auch aus dem Arabischen übernommen worden. Die daraus resultierenden Sprachen werden heute Urdu und Hindustani genannt. Hier seien ferner das Bengali, Bihari, Assami, das Hindi und Gudscherati sowie das Radschasthani als bedeutende, im Norden zu findende und zum arischen Prakrit-Kreis zu rechnende Sprachen mit persisch-arabischem Einfluß erwähnt.

In Indien unterscheidet man zwei Arten gelehrter ${ }^{2}$, im Lesen und Schreiben bewanderter Ärzte: die Väidjas und Hakiems. Sie sind sowohl ihrer Religion als auch ihrer Schriftart und ihres medizinischen Systems nach voneinander verschieden. Der Väidja ist indischen Glaubens, spricht eine oder mehrere indische Sprachen und kennt einen Teil der indischen medizinischen Literatur. Sein wissenschaftliches System nennt sich ajurvedisches System. Der Hakiem dagegen ist Mohammedaner. Er braucht die arabischen Schriftzeichen, spricht Urdu, Hindustani und Persisch und nennt sein medizinisches System Junani-System. Dieser Name weist schon auf den ionischen, d.h. griechischen Ursprung seiner Heilkunde hin. Sie ist über Arabien und Persien nach Indien gelangt. Die indischen Herrscher, sowohl die mohammedanischen wie auch die heidnischen, waren meist tolerant gegen die andersgläubigen Ärzte.

Während der Hakiem als Mohammedaner abseits von der indischen Kastengliederung stand, hatte sich der Väidja in die Kastenordnung zu fügen; vor allem hatte er sich mit der höchstangesehenen Kaste, mit den Brahmanen, auseinanderzusetzen; denn die Brahmanen betrieben neben der philosophischen, theologischen und juristischen auch die medizinische Wissenschaft. Hier ist allerdings bemerkenswert, daß die Brahmanen nur in

${ }^{2}$ Unter einem ungebildeten, in Lesen und Schreiben nicht bewanderten Arzt hat man den Heilkundigen zu verstehen, der beispielsweise auf den Straßen der Großstädte sitzt, Wurzeln und andere Drogen auf einer Decke ausgebreitet zum Verkauf anbietet, flüchtige Untersuchungen vornimmt, Diagnosen stellt und vor allem Therapie treibt. Für einen solchen "Kurpfuscher» oder "Quacksalber» hat man, genau wie bei uns, zahlreiche Bezeichnungen. Wohlwollend wird er «niem Hakiem» genannt, was halbgebildeter Arzt heißt. Ein Sprichwort sagt: «Niem Hakiem, Khatraa e Dschaan», zu deutsch: «Ein halbgebildeter Arzt (ist) eine Gefahr für das Leben.» 
seltenen Ausnahmen selbst ärztliche Praxis übten (4). Sie zogen es vor zu theoretisieren, wie es zahlreichen alten Reiseberichten zu entnehmen ist. Die medizinische Praxis überließen sie dem Väidja. Die Väidjas bildeten in Bengalen, Assam und einigen angrenzenden Gebieten eine eigene Kaste; in den übrigen Teilen Indiens gehörten sie zu der dritten Kaste, den Sudras, in denen sie sich zu den Handwerkern, Fischern usw. gesellten. Die Kaste der Väidjas nennt den Nordosten Indiens als ihre Heimat, und tatsächlich findet man die Väidjas in Bengalen in sehr großer Zahl. Hier sollen sie ihrer Überlieferung nach einstmals auch einige Könige gestellt haben. In Bengalen ist aber nun nicht jeder Kasten-Väidja gleich auch ein Arzt. Ein Angehöriger dieser Kaste kann durchaus einem andern bürgerlichen Beruf nachgehen. Die Verhältnisse haben sich hier seit dem vorigen Jahrhundert sehr gelockert! Die Kaste der Väidjas folgt an Ansehen gleich hinter der der Brahmanen. Den Brahmanen ist aber eigen, Angehörige einer andern Kaste nicht in ihre Sanskrit-Bücher, «nicht in ihre Karten» schauen zu lassen. Hiervon wird lediglich den Väidjas eine Ausnahme gewährt. Sie dürfen Sanskrit lernen und Einblick in die Bücher der Pandits tun. Die Väidjas haben außerdem auch ihre eigene Literatur. Im Laufe der Geschichte konnten sie immer wieder ihre eigenen Erfahrungen niederschreiben und taten das nicht nur in Sanskrit, sondern auch in den Sprachen der Länder, in denen sie Praxis übten.

\section{Portugiesische Zeit}

In die eben genannte indische Sprachwelt stießen nun die Europäer, zunächst die Portugiesen. Ihre Fahrten nach Indien galten den Schätzen des Landes: den Drogen, Gewürzen und Heilpflanzen. Schon früh sehen wir daher in Indien portugiesische Apotheker und Botaniker (5). Sie waren nicht nur an den Apotheken der sogleich errichteten Hospitäler tätig, sondern hatten auch die Erforschung der indischen Heilpflanzen zur Aufgabe. Sie betrieben Pharmakognosie und brachten den Handel in Gang.

In Spanien und Portugal hörte man sagenhafte Dinge vom Reichtum der neu entdeckten Erdteile, und besonders begeisterte sich die Jugend für die fernen Länder. Zu dieser Zeit studierte ein junger portugiesischer christlicher Jude, Garcia dA ORTA, Geographie und Botanik; später studierte er Medizin. 1534 reiste er als Arzt nach Indien. Achtundzwanzig Jahre blieb er dort und ließ sich Zeit, das Land gründlich zu studieren, reiste umher und behandelte Patienten, Europäer und Inder, unter letzteren auch manch einen 
Fürsten. Garcia da Orta erforschte die indischen Krankheiten, baute in Bombay einen botanischen Garten und gewann Hakiems und Väidjas zur wissenschaftlichen Diskussion. Schließlich ging er daran, seine Erfahrungen niederzuschreiben, und sein Buch konnte in Goa gedruckt werden. Im Jahre 1563 erschien es unter dem Titel Colloquios dos simples e drogas e cousas medicinais da India. Als Setzer zeichnet ein Deutscher, Joanives de Endem. Das Buch ist heute vor allem durch die Beschreibung der Heilpflanze Rauwolfia serpentina und der Choleraepidemien bekannt.

Hier interessiert uns an dem Buch allerdings etwas anderes: Es vermittelt einen Eindruck von den sprachlichen Kenntnissen seines Autors. Diese dürften nicht zu tief gewesen sein, weshalb er möglichst viele Begriffe portugiesisch brachte. So spricht er von fisicos indianos und fisicos arabos statt von Väidjas und Hakiems. Zusammenfassend nannte er beide fisicos gentios.

Garcia da Orta verfaßte sein Buch als Kolloquium. Ein Arzt namens Ruano stellt die Fragen, andere Personen, so z.B. Diener oder Patienten, können in die Unterhaltung eingreifen, und Garcia da Orta antwortet. Während einer Unterhaltung zwischen Ruano und Orta ereignete es sich einmal, daß eine Dienerin hereinkam und meldete: ein Junge sei draußen. Dieser berichtet: Er komme von Don Geronimo. Einer von dessen Brüdern sei erkrankt. Der Doktor möge sobald wie möglich kommen. Auf die Frage von Orta antwortet der Junge mit portugiesischen Worten, daß der Patient von der «morxi» befallen sei. Das Wort morxi ist indisch. Noch ehe Orta zum Kranken eilt, erklärt er Ruano, was man unter «morxi» in Indien versteht (6). Es sei die «colerico passio», die Cholera, welche die Inder «morxi» nennen. Daraus hätten die Portugiesen das Wort mordexi gebildet. Die Araber würden die Cholera «hachaiza» nennen (7).

Wie vollzog sich nun das Befragen eines indischen Arztes? Hiervon vermittelt Garcia da Orta ebenfalls ein Bild (8). In ihrer Unterhaltung kommt Ruano auf das indische Turbit ${ }^{3}$ zu sprechen. Er möchte von Orta Auskunft haben, wie die Inder dieses Abführmittel (9) anwenden. Orta rät, hierüber am besten einen indischen Arzt aus Goa zu fragen, der gerade in der Nähe ist. Und schon ruft die Dienerin nach Malupa, dem eingeborenen Arzt, der jeden Morgen die Schwarzen behandelt. Malupa kommt und

${ }^{3}$ Schon vor Garcia da Orta hat ein anderer Portugiese, der Apotheker TomÉ Pires, über das indische Turbit berichtet. Er schrieb am 27.Januar 1516 aus Kotschin einen Brief «Über die Drogen und wie sie wachsen» an seinen König, worin verschiedene Turbitarten diskutiert werden (zit. nach: The Suma Oriental of Tomé Pires, Band 2, London 1944, S. 512-517). 
berichtet gern. Dabei spricht er ebenfalls portugiesisch. Er vergleicht das Turbit mit dem indischen Ingwer, einer Droge gegen Verdauungsstörungen (10).

Die Unterhaltung mit dem goanesischen Väidja Malupa wirft die Frage auf, welche Stellung die einheimischen Ärzte zu den europäischen hatten. Hier läßt sich einiges schon aus der gesamten Situation sagen: Es war das Ziel der Portugiesen, möglichst weite Teile der Küsten Afrikas, Asiens und Indonesiens unter ihren Einfluß zu bekommen. Besonders in Indien erzielten sie in dieser Hinsicht große Erfolge. Hier ließen sich mehrere hunderttausend Menschen christianisieren, erhielten portugiesische Namen und lernten die portugiesische Sprache. Das 1511 eroberte Goa wurde zur Hauptstadt von Asien und zum Sitz des Vizekönigs erhoben. Anfang des 17.Jahrhunderts zählte es an die 250000 Einwohner. Noch heute zeugen stolze Ruinen vom Glanz der einstigen Weltstadt. Portugal selbst hatte zu dieser Zeit kaum mehr als eine Million Einwohner. Das Mutterland konnte also auf die Dauer gar nicht die Menschen aufbringen, die in den überseeischen Besitzungen verschlungen wurden. Es war viel zu schwach, das Weltreich allein zu beherrschen, und bedurfte daher der Mithilfe der eingegliederten fremden Völker. Aber wie sollte man diese zur Mithilfe gewinnen?

Vielfältig voneinander verschiedene Menschen zusammenzuhalten, kann möglich werden, wenn man ihnen die Idee, gemeinsam ein großes Reich zu schaffen, vorzeichnet und wenn man sie im Hinblick auf das Kreuz Christi menschenwürdig behandelt und ihnen in rechtlicher Hinsicht Gleichstellung gewährt. Doch dem Rechner gelten diese philanthropen Ideen nicht viel; er sieht mehr den Handel, der unter dem Schutz einer militärischen Macht, hier der Portugiesen, an den Küsten und über das Meer geführt werden kann und gute Geschäfte bringt. Alles dieses mögen die Portugiesen, unter ihnen besonders der große Vizekönig und Reichsgründer Albuquerque, durchdacht haben. Goa sollte also zum Fanal eines solchen Reiches werden. Hier kamen Christen, Moslems, Juden und Hindus, Europäer, Araber, Chinesen, die Vertreter der verschiedensten indischen Völker, Leute aus Malakka und Java, aus Mozambique und Sansibar zusammen, wickelten ihre Handelsgeschäfte ab oder ließen sich gar nieder.

Goa wurde durch den Vizekönig Albuquerque den Moslems abgenommen. Die Inder, bisher wirtschaftlich von den islamischen Herrschern unterdrückt, feierten den Portugiesen als ihren Befreier. Albuquerque ist der bedeutendste unter den Begründern des Portugiesisch-asiatischen Reiches, und er trat schon früh für eine möglichst große Gleichberechtigung der Europäer und Inder ein. Mischehen als äußeres Zeichen dieser Politik wurden besonders gefördert. Aus den verschiedensten Teilen der Welt kamen Händler, Handwerker Künstler und Soldaten nach Goa. Die Stadt wuchs schnell und wurde reich. Um dem Gewirr der Sprachen ein Ende zu bereiten, zogen die in den verschiedensten Gegenden beheimateten Fremden es vor, Portugiesisch zu sprechen; es wurde zur Staatssprache. Man verstand es von der persischen Küste bis zu den Molukken. 
Goa sollte das Zentrum der Christenheit in Asien werden. Franziskus Xaverius eilte dorthin, um die zusammenlaufenden Menschenmassen zu christianisieren. Wollte man den Missionserfolg sichern, so war dazu auch christliche Literatur nötig. Die Jesuiten brachten also auch Druckpressen nach Indien, mit denen man gleich an Ort und Stelle christliches Schrifttum vervielfältigen konnte; es waren die ersten Indiens. Der Papst ernannte den Bischof von Goa zum Erzbischof von Asien. Sein Bistum reichte von Kapstadt bis Japan. In Goa wie in andern Teilen Portugiesisch-Asiens entstanden imposante Kirchen, von denen sich einige der heute noch erhaltenen Kirchen durchaus mit bedeutenden Barockkirchen Deutschlands vergleichen lassen. Gewaltige Festungsanlagen, Paläste und Klöster und nicht zuletzt eine große Zahl Hospitäler wurden an den verschiedensten Orten errichtet. Mit der Zeit entstanden auch erste Gemäldegalerien und Bibliotheken.

Schon Garcia da Orta benutzte verschiedene Bücher. So zitiert er in seinem berühmten Werk einige Autoren, darunter Vesalius, dessen De humani corporis fabrica 1543 erschien. Er nennt weiterhin Amato LusiTANO, der über die Materia medica des DIOsKorides schrieb (veröffentlicht in Venedig 1553). Außerdem erwähnt er Johannes Manardus und seine medizinischen Briefe, die 1540 in Basel veröffentlicht wurden, sowie die Anmerkungen zur Materia medica des Dioskorides von Valerius Cordus, die Conrad Gessner mit dem gesamten Nachlaß des Cordus 1561 in Straßburg herausbrachte (11). Da Garcia da Orta schon 1534 nach Indien abgereist war, kann er erst in Indien Kenntnis von diesen Männern und ihren Büchern erhalten haben.

In Goa entstand gegen Ende des 16.Jahrhunderts nach zeitgenössischen Berichten das «vortrefflichste» Hospital der Welt. Auch in militärischer Hinsicht sowie in Handel und Gewerbe kam es zu bedeutender Entwicklung. Schon waren Ansehen und Macht der Portugiesen in Asien so stark gewachsen, daß der König in der Heimat mißtrauisch auf seinen fern weilenden Vizekönig wurde, der mehr Menschen und ein vielfach größeres und reicheres Land als Portugal beherrschte. Deshalb bestimmte er, daß jeder Vizekönig nach drei Jahren abgelöst werden mußte. Der Papst sandte zur Überwachung der eigenwillig werdenden Christen und christlichen Institutionen die Inquisition.

In dieser Welt standen sich also die einheimischen und indischen Ärzte gegenüber. Bereits Albuquerque scheint eine erste Lösung für ihr Verhältnis zueinander gefunden zu haben; denn wir hören, daß ihm auf seinen Heerzügen neben den europäischen Ärzten und Chirurgen auch Väidjas dienten. Seit dieser frühen Zeit besitzen die Väidjas bei den Portugiesen in Indien einiges fachliches Ansehen (12). 
Die Respektierung gewisser heilkundlicher Fähigkeiten der Väidjas blieb in den portugiesischen Besitzungen übrigens bis zum Ende der Kolonie. Während meines Besuches in Indien 1961 fand ich in Pangim, in der Hauptstadt des Landes Goa, eine große Hindu-Apotheke, die in einem zentralgelegenen, repräsentativen Gebäude untergebracht war. Ihr Leiter war ein Väidja. Sein Hauptinteresse war es, Medizinalpflanzen nach indischen Rezepten zu verarbeiten und zu verkaufen. Daneben hatte er auch ein modernes Gerät für Elektrokardiographie.

Als die Nehru-Truppen schließlich in die portugiesisch-indische Provinz Goa einrückten, fanden sie in Ponda, einer andern Stadt, wie Goanesen und Portugiesen gerade dabei waren, auf dem neuen Marktplatz ein Denkmal für den Dada-Väidja zu errichten. Dieser einheimische Arzt hatte sich durch seine vielfältigen Erfolge einen Namen geschaffen. Er kannte sich nicht nur in der verschiedenartigen alten indischen Literatur aus, sondern verfaßte auch selbst medizinische Werke. Er war mit der Zeit reich geworden, verwandte aber sein Vermögen zu sozialen Zwecken, zum Bau von Wohnblocks und Schulen sowie zur Fürsorge für arme Leute.

Heute können alle gelehrten Väidjas in Indien entweder Englisch oder Portugiesisch. Von 1511 bis 1650 war das Portugiesische an den Küsten Asiens die vorherrschende Sprache. Es wundert uns daher nicht, wenn Garcia da Orta mit seinem Väidja portugiesisch spricht. Garcia erhielt so Kenntnis von der indischen Medizin in seiner Landessprache. Natürlich wird er auch einige Worte oder sogar ganze Sätze der einen oder andern indischen Sprache gelernt haben. Ob er die fremde Schrift lesen konnte, ist schon fraglich. Ein eingehendes Studium der indischen Literatur dürfen wir bei ihm also nicht erwarten. Man muß sich die Forschungsweise Garcia da Ortas wie folgt vorstellen: Es war bekannt, daß er sich für indische Pflanzen interessierte, und so brachte man ihm welche. Auch wird er die Praxis des einen oder andern Väidjas besucht, sie bei der Arbeit beobachtet und einen Einblick in ihre Drogensammlungen getan haben. Die Väidjas gaben ihm dann entweder in portugiesischer Sprache oder über Dolmetscher die entsprechenden Erklärungen.

Wie schon angedeutet, hatte Garcia eine beachtliche Bibliothek zur Hand. Hier konnte er bereits Bekanntes heraussuchen und zur Diskussion heranziehen. Das Hauptinteresse nicht nur der Apotheker, sondern auch der Ärzte gehörte vor allem den Heilpflanzen; denn die Inder boten auf andern Gebieten der Pharmazie und der übrigen Medizin nicht viel Neues. Zur genauen Beobachtung der Pflanzen legte man botanische Gärten an, bei- 
spielsweise in Goa. Garcia da Orta besaß einen eigenen Garten auf der Insel Bombay, die er vom König von Portugal als Geschenk erhalten hatte.

Das Werk Garcias nennt eine Vielzahl an Völkern und Ländern mit den dort üblichen Beschreibungen und Namen von Heilpflanzen; so nennt es die Türken, Armenier und Perser, die Mauren, Äthiopier und Araber, die Kanarinen, Dekkaninen, Malabaren, Maldivier, die Bengalen, Gudscheraten (Baneanen) und Brahmanen. Es wird Usbekistan, Ceylon, Siam, Malakka, Borneo, Java, Timor und China erwähnt. Als Herkunftsorte bzw. Handelsstädte findet man Konstantinopel, Beirut, Jerusalem, Alexandrien, Kairo, Ormuz vor Persien, Kalikut, Schaul, Kotschin und Kranganor, Dely und Kambaja, neben Goa, Diu usw. Autoren wie Dioskorides, Plinius, Antonius Musa und Galen, Avicenna und Rhazes ergänzen die vielfältige Übersicht. Kurz, Garcia entwirft ein umfássendes Bild von der orientalischen Pharmakogeographie.

Es versteht sich, daß Garcia nicht alle diese Völker, Länder und Städte besucht hat. Er wird, wie schon angedeutet, manche Einzelheiten der Literatur entnommen haben. Zum Studium der orientalischen Literatur hat er Dolmetscher benutzt. J. Gerson da CunHa wies bereits 1900 darauf hin, daß der persische Interpret Kwajeh Parkuli Garcia in Bassein türkische, persische und arabische Namen nannte (13).

Zwanzig Jahre nach der Publikation Garcias (1563) kommt ein Florentiner Kaufmann, Filippo Sassetri (geb. 1540) nach Indien. Auch er ist bestrebt, Einblick in die indische Pharmazie zu erhalten. Er wird schon vorher das Werk Garcias und die Kritik daran studiert haben. Er geht jedenfalls ganz anders vor als Garcia und beschränkt sich auf ein indisches Wörterbuch, das er mit Hilfe eines eingeborenen Arztes übersetzt. Leider ist sein Manuskript nicht zum Druck gekommen, und so hat es wohl kaum Einfluß gewinnen können. Das Unternehmen Sassettis ist lediglich aus einem Brief bekannt, den er an den Granduca di Toscana sandte. Sassetti ist 1588 in Indien, vermutlich in Goa gestorben, und so ist sein Werk nicht mehr gedruckt worden (14).

\section{Holländische Zeit}

Hundert Jahre währte die portugiesische Alleinherrschaft über Indiens Küsten. Dann, um 1600, tauchten andere europäische Nationen als Konkurrenten auf. Unter ihnen gewannen schließlich die Holländer für einige Zeit die Vorherrschaft. Auch sie bekundeten großes Interesse an der Er- 
forschung Indiens, besonders der indischen Botanik ${ }^{4}$; denn auch ihnen versprach der Handel mit Drogen große Geschäfte. Zur holländischen Zeit erschienen viele Bücher über Indien, so eins von dem Arzt Jаков Bontius, 1598 in Leiden geboren, der wichtige Einzelheiten über die indische Medizin aufgeschrieben hat. Er war 1627 als Inspektor der Chirurgen nach Indien gekommen und trug hier innerhalb von vier Jahren - er starb bereits 1631 ein großes Material zusammen. Sein Name ist vor allem durch die Beschreibung der Beriberi bekannt geworden. Ähnlich wie Garcia hat auch Bontius eine große Zahl Heilpflanzen erwähnt (15). Allein, es fehlten auch ihm die Sprachkenntnisse. So hat er ähnlich wie Garcia arbeiten müssen. In philologischer Hinsicht blieb also eine große Lücke weiterhin bestehen.

Über vierzig Jahre später beginnen zwei Leute in Holländisch-Indien mit der systematischen Erforschung der Heilpflanzen. Einer war der Deutsche Paul Hermann (geb. zu Halle 1640), der 1672 als Arzt nach Ceylon kam. Er stieg zum Chefarzt auf und wurde Oberinspektor des Hospitals von Colombo (16), widmete sich aber vornehmlich botanischen Studien. Er legte zwei Herbarien an, die später nach London ins Britische Museum und ins Rijksherbarium nach Leiden gelangten und von denen noch heute große Teile erhalten sind $(17,18)$. Etwa um die gleiche Zeit wie Hermann beginnt auch der Gouverneur von Holländisch-Malabar, Hendrik van Rheede van Drakenstein, mit der systematischen Erforschung und Beschreibung der in dieser Gegend Indiens zu findenden Pflanzen, besonders der Heilpflanzen.

Beide Sammler haben in ihren Schriften neben die Pflanzen oder Zeichnungen außer den lateinischen Namen auch die indischen Namen geschrieben. Es fällt auf, daß die letzteren in der einheimischen Schrift vermerkt sind. So zeigen die zwei in Leiden erhaltenen Bände des Hermannschen Herbarius vivus mit der Hand geschriebene ceylonesische Worte (Singha-

${ }^{4}$ Anfang des 17. Jahrhunderts bekundeten die Engländer, Holländer, Franzosen und Dänen ihr Interesse an Indien, indem sie ostindische Kompanien gründeten oder deren Gründung anstrebten. Vorher waren schon verschiedene Leute aus diesen Ländern zu Land oder mit den Portugiesen über See nach Indien gefahren und hatten Einblick in die dortigen Verhältnisse gewonnen. Es sei die Reise des Jean MoQvet genannt, der 1601 erstmalig gestartet war, aber erst 1607 erfolgreich nach Indien gelangte. Er war Hofapotheker des französischen Königs, und so erhielt er in Goa eine Anstellung im großen Hospital. Quartier bezog er bei einem indischen Chirurgen, der Antonio Fernandes hieß. Dessen Frau war eine Chinesin, ein Beispiel für das Beieinander der verschiedenen Nationen in der Weltstadt Goa! (Jean Moqvet, Voyage en Afrique, Asie, Indes Orientales et Occidentales, Paris 1617, S. 274f. und 306). 
lesisch und Tamil), neben Jasminum pennatum das ceylonesische Wort Bambu. Bei Hendrik van Rheede können wir sein vielbändiges Prachtwerk Hortus Indicus Malabaricus heranziehen. Der erste Band erschien 1678 in Amsterdam. Neben den herrlichen gedruckten Pflanzenbildern folgt unter dem lateinischen Namen der malabarische, der arabische und der brahmanische Name jeweils in der entsprechenden Schrift.

Beherrschten Hermann und van Rheede diese Sprachen? Wir wissen es nicht; doch so viel können wir sagen, sie haben sich jedenfalls bemüht, die Lücke hinsichtlich der Nomenklatur zu schließen! Beginnen wir mit der Forschungsmethode van Rheedes: Als Gouverneur in Malabar stellte er eine Forschungsgemeinschaft zusammen, die 1674 in Kotschin mit der Forschung anfing. Zu ihm gehörten der Geistliche Johannes Caesarius aus Kotschin, der Väidja (Doctor Malabaricus) Itті Achudem Coladda aus Karrastadt (Karrapuram), von dem es ausdrücklich heißt, daß seine Vorfahren schon Väidjas waren (Abb. 1 a), und Emanuel Carneiro, ein römisch-christlicher Einwohner aus Kotschin, der bei der holländischen Ostindien-Kompanie als Dolmetscher vereidigt war. Carneiro hatte die Aufgabe, die Bezeichnungen für die Bäume, Sträucher, Blätter und Blüten sowie deren medizinische Wirkungen, die Coladda in malabarischer Sprache angab, ins Portugiesische zu übersetzen.

Carneiro trägt einen portugiesischen Namen; doch vermutlich war er kein Portugiese, sondern bestenfalls ein Mestize. Hierzu sei gesagt, daß Kotschin bis 1662 portugiesisch war. In diesem Jahr wurde es durch die Holländer nach harter Auseinandersetzung erobert. Die Reste der portugiesischen Streitkräfte erhielten nach der Kapitulation einen ehrenvollen Abzug. Von ihnen blieben in der Stadt lediglich die Kranken und Verwundeten zurück. Die Zivilbevölkerung konnte sich Holland unterwerfen. Wer es tat, durfte wohnen bleiben, seine katholische Religion beibehalten und frei Handel üben. Franziskaner versahen den Gottesdienst (18).

Von den sogenannten Portugiesen blieben in den holländisch gewordenen Städten meist nur die zurück, die dort enge heimatliche Bindungen hatten, so die Mestizen und vor allem auch die Inder, welche den römischen Glauben und portugiesische Namen angenommen hatten. Da Carneiro außer dem Portugiesischen auch das Malabarische (Tamil, vielleicht auch Malajalam ?) beherrschte und nur wenige Portugiesen das Malabarische lernten, sehen wir hierin eine Bekräftigung der Vermutung, daß er kein reiner Portugiese war. Daß Carneiro nicht nur malabarisch lesen, sondern auch schreiben konnte, bekundet er durch einen von ihm unterzeichneten, in den (südlichen) 


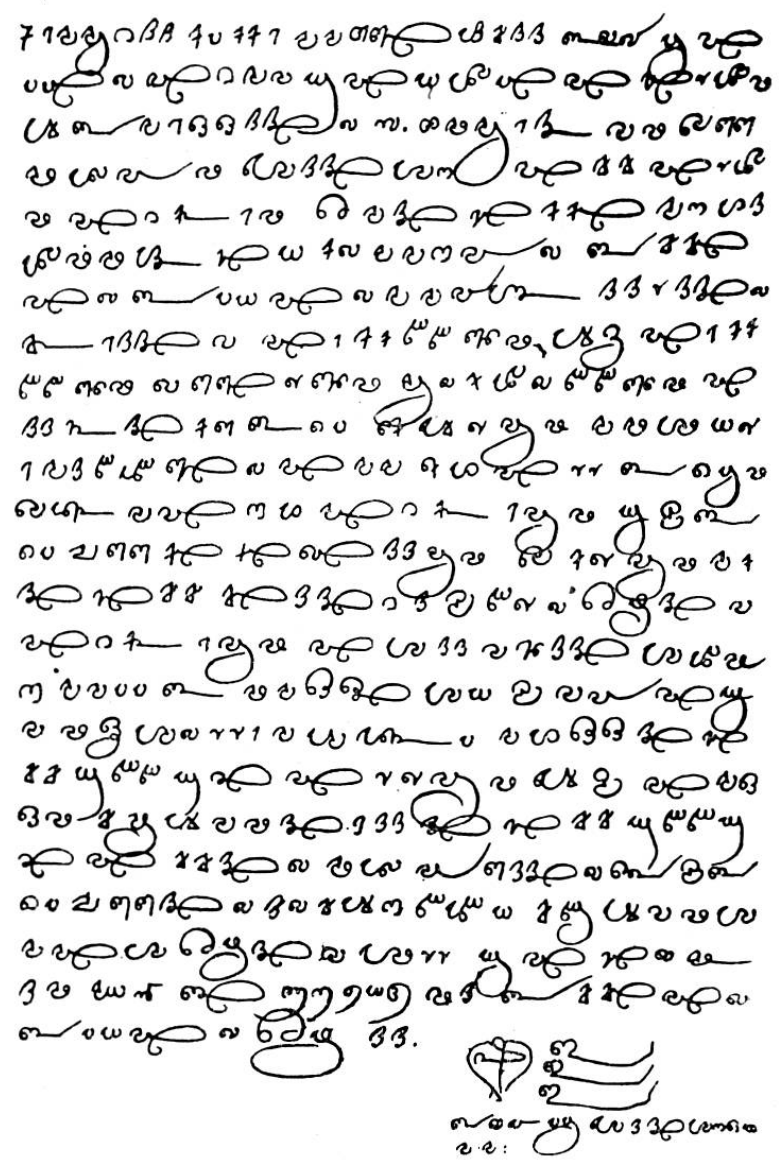

\title{
HOC E ST.
}

$\mathbf{E}$

Go IT I I ACHUDEM Doctor Malabaricus Natione Chego, gentilis \& naturalis in Carrapuràm, feu terra dicta Codda Carapalli, habitator ædium dictarum Coladda, qui proavis, avis \& parentibus Medicis feu Doctoribus natus fum, teftor me per mandatum D. Commendatoris HENRICI a RHEEDE, veniffe in Civitatem Cochinenfem \& per EMANUELEM CARNEIRO Nobilis Societatis Indic $\mathfrak{x}$ interpretem dixiffe \& dictâffe nomina, virtutes medicas \& proprietates arborum, plantarum, herbanum \&

$\star \star \star \star$ con-

\begin{abstract}
Abb. 1 a. Handschrift des malabarischen Arztes Itti Achudem Coladda (aus Hendrik van Rheede, Hortus Indicus Malabaricus, Amsterdam 1678). B. Ziegenbalg vermerkt in seiner 1716 in Halle erschienenen Grammatica Damulica, daß die hier angeführte malabarische Schrift (Tamil) in vieler Hinsicht von den üblichen malabarischen Schriftzeichen abweiche und selbst den von ihm befragten Indern unbekannt gewesen sei. Vielleicht handelt es sich hier um eine altertümliche Schreibweise des Malajalam, welche Sprache früher zur Lingua malabarica hinzugerechnet wurde
\end{abstract}




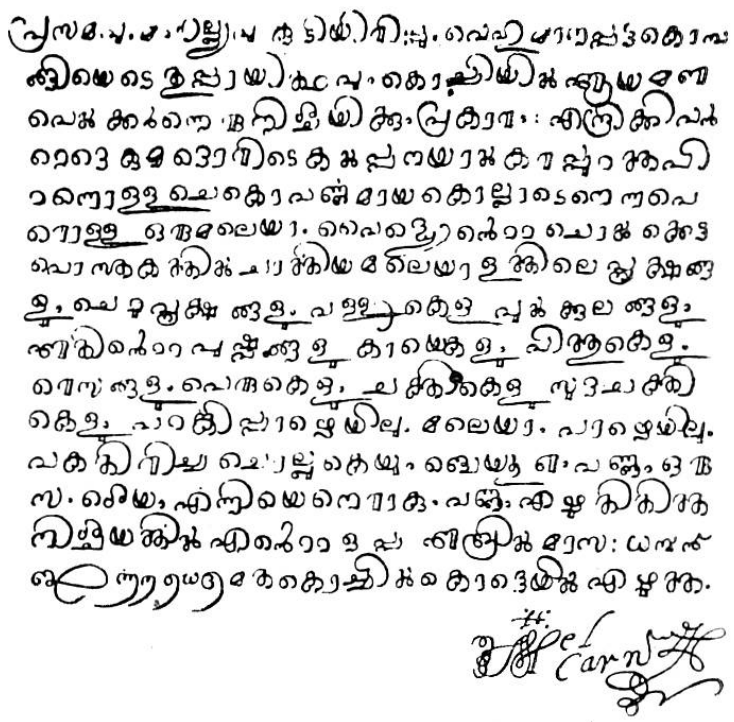

\section{$\begin{array}{llllll}H & O & C & E & S & T \text {. }\end{array}$}

7 Go Emanuel Carneiro, Chrinenfis, interpres juratus N. Societatis Indica per mandatum $\mathrm{D}$. Commendatoris $\mathrm{HEN-}$ RICI à RHEEDE ex lingua Malabarica in lingua Lufitanica explicavi \& interpretatus fum nomina, virtutes medicas, \& proprie. tates arborum, plantarum, herbarum \& convolvulorum nafcentium in hifce terris Malabaricis, auditas à CoL A D D D Doctore Malabarico gentili, \& Natione Cbeguo naturali in Carruparàm, ac in Doctorum Malabarico-

Abb. 1 b. Handschrift des vereidigten Dolmetschers der holländischen Ostindien-Kompanie Emanuel Carneiro (aus Hortus Indicus Malabaricus, 1678)

Schriftzeichen des Tamil geschriebenen Text, den van Rheede in seinem Buch zum Abdruck gebracht hat. (Abb. 1b).

Der Gemeinschaft gehörten ferner drei Brahmanen an, die ihre Namen in der lateinischen Schrift Ranga Botto, Vinaique Pandito und Apu Вотто schrieben. Sie sammelten in Kotschin ebenfalls Pflanzen, Blätter, Blüten und Früchte. Bei ihrer Arbeit zogen sie auch eins ihrer Sanskrit- 


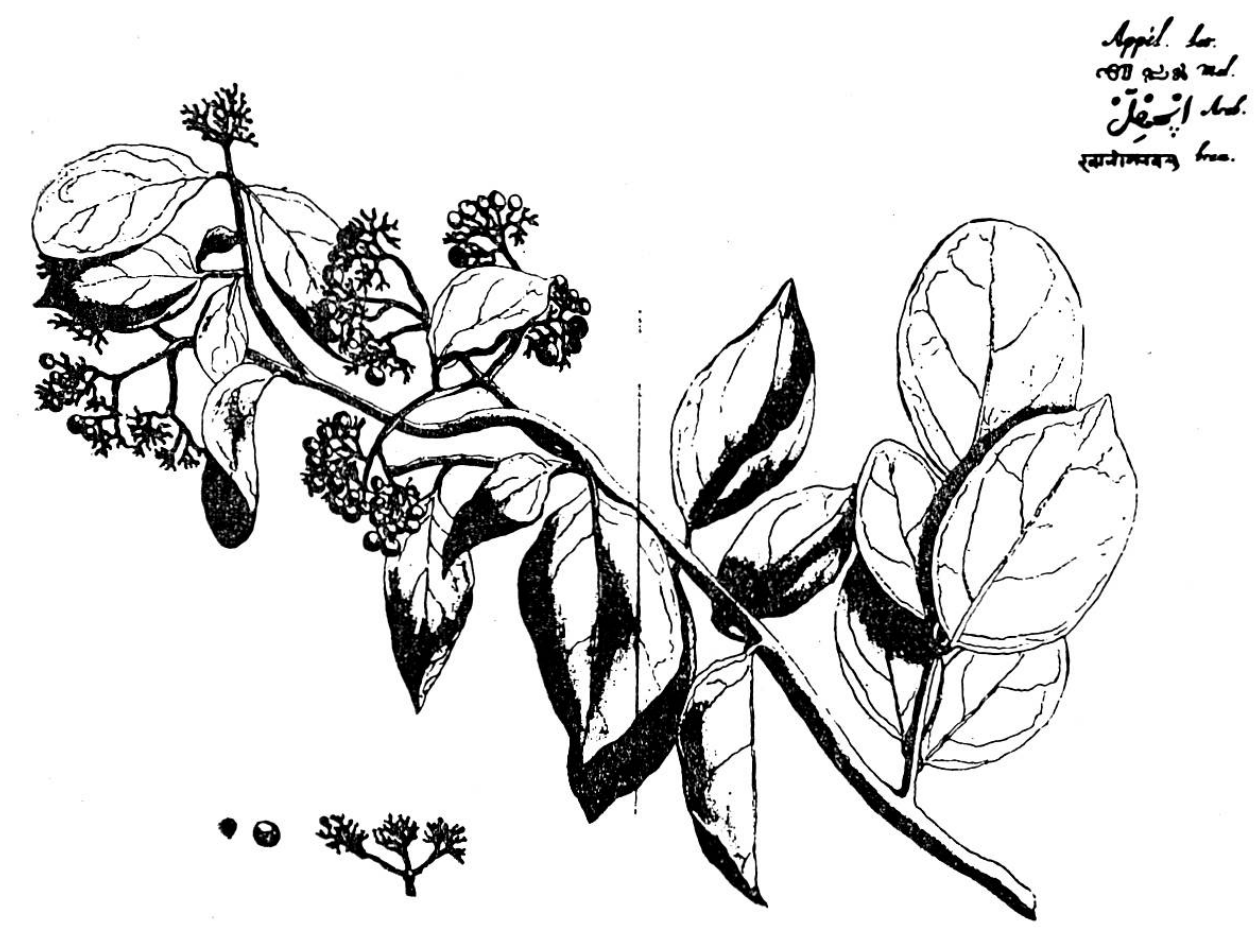

Abb. 2 a. Eine Pflanze, Appél, aus Hortus Indicus Malabaricus, Band 1, 1678, S. 53

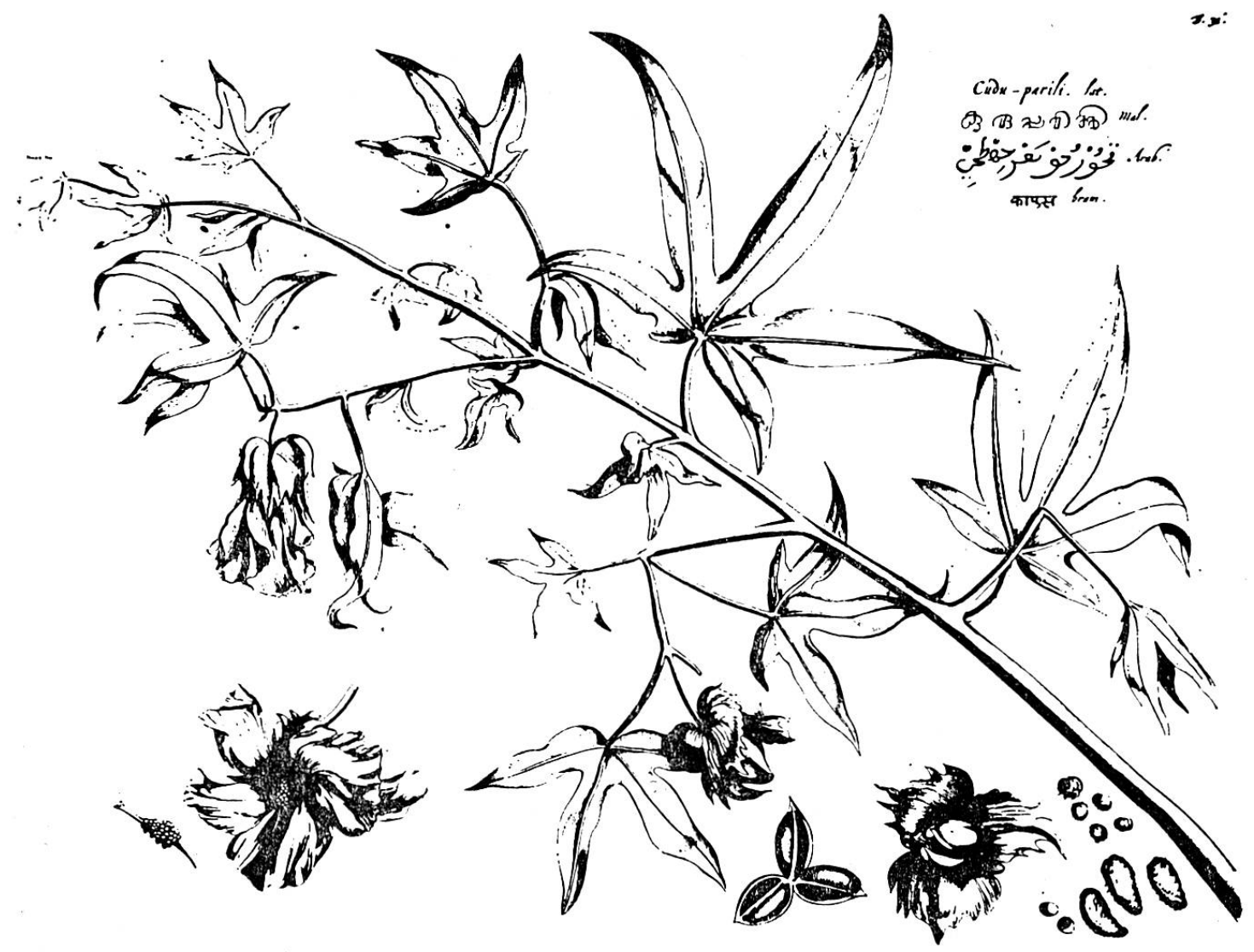

Abb. 2b. Eine andere Pflanze, Cadu-pariti, aus Hortus Indicus Malabaricus, Band 1, 1678, S. 56. Ihre Blätter wurden zur Herstellung eines Medikamentes gegen Schmerzen, besonders gegen Kopfschmerzen, und deren Früchte gegen Dysenterie verwandt 


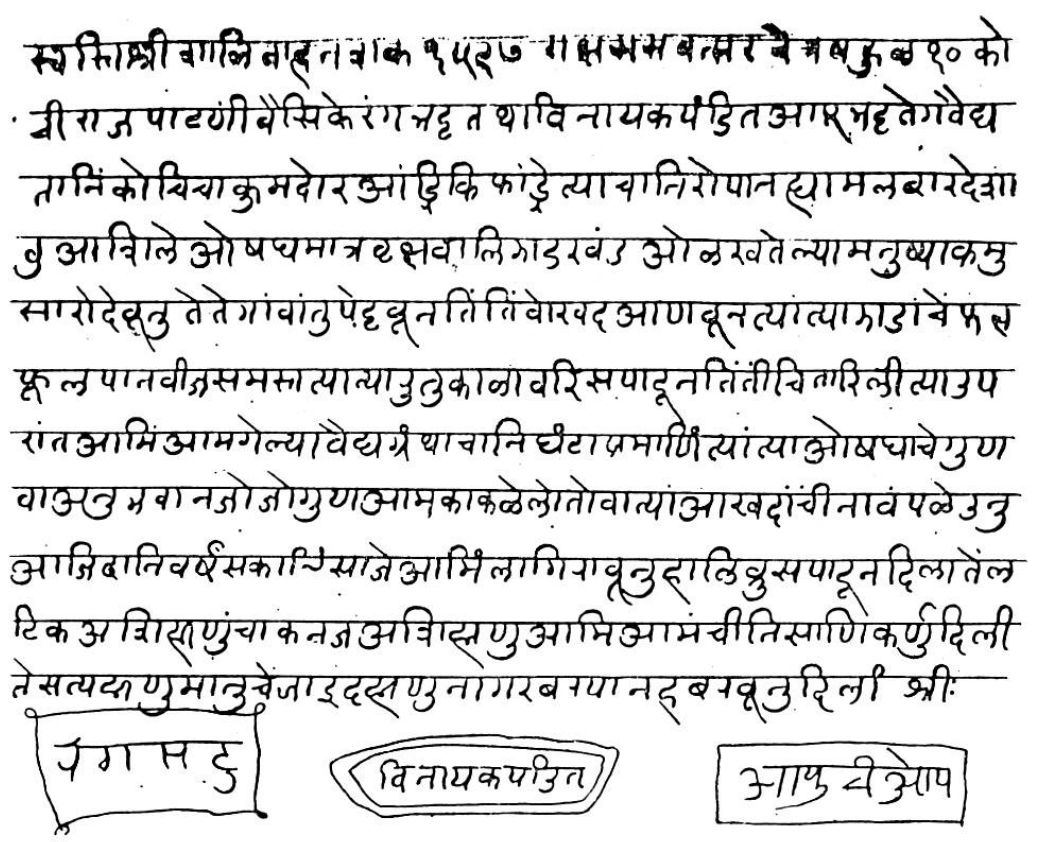

$$
\text { HOC }{ }_{\vee} S T \text {. }
$$

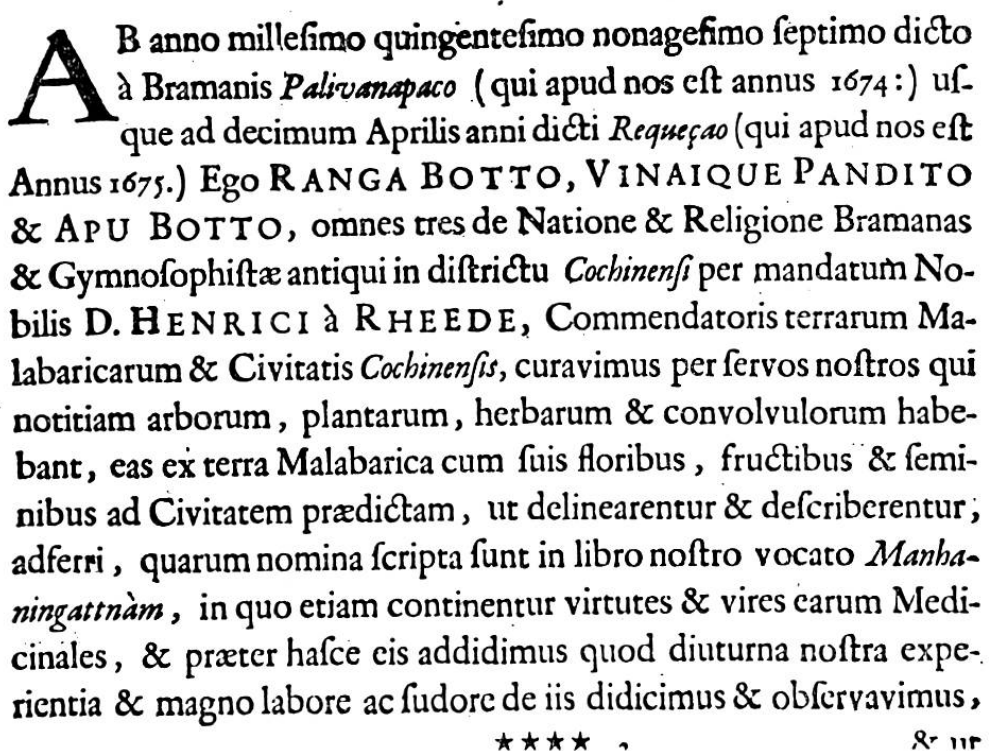

Abb. 3 a. Handschriftlicher Bericht der drei Brahmanen, die Hendrik van Rheede bei der Zusammenstellung indischer Pflanzen und bei der Übersetzung von Sanskrit-Worten halfen (aus Hortus Indicus Malabaricus, 1678). Der Sanskrit-Text ist im Devanagari geschrieben. Herr Professor Losch hat die hier von den Holländern im Lateintext als Ranga Botto, Vinaique Pandito und Apu Botto geschriebenen Namen aus dem Devanagari in unsere heutige Transskriptionsweise umgeschrieben: Rangabhațta, Vinājaka-Pandita und Āpūbhațța 
Bücher zu Hilfe, das Mahaningattnam hieß. Ihm entnahmen sie Namen, Aussehen und Wirkungen von Medizinalpflanzen. Wenn wir im Hortus Indicus Malabaricus auf den Bildtafeln neben den Pflanzen «brahmanische» Namen finden (Abb. $2 \mathrm{a}$ und $2 \mathrm{~b}$ ), so stammen sie vermutlich alle von den drei zuletzt genannten Gelehrten. Hendrik van Rheede würdigte ihre Leistungen dadurch, daß er einen von den Brahmanen verfaßten SanskritText in den ersten Band seines Werkes aufnahm. Er ist, ähnlich wie der Tamil-Text, von jedem der drei Brahmanen unterschrieben (Abb. 3 a). Hier sei kurz eingeschoben, daß der Sanskrit-Text in der Schrift Devanagari, was «göttliches Nagari» heißt, geschrieben ist (Abb. 3 b). Er kam als Block zum Abdruck. Druckhistorisch ist dabei interessant, daß es sich um den ersten Druck dieser Schrift handelt (19).

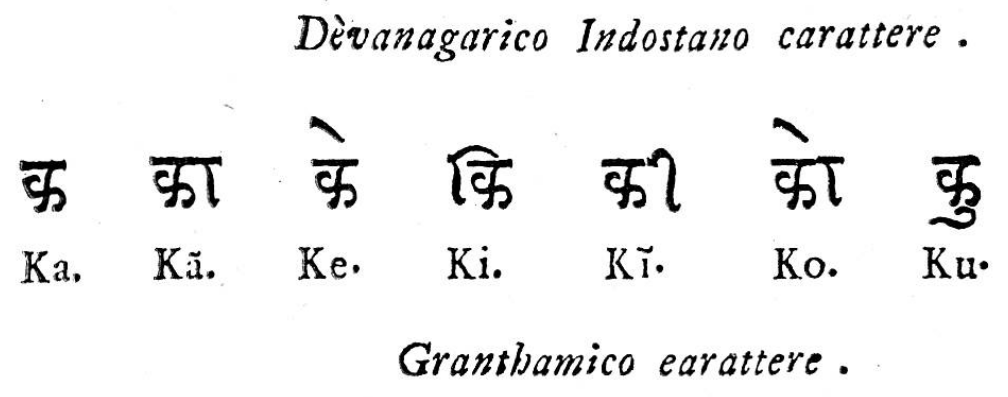

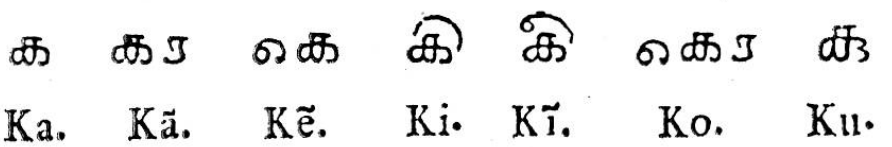

Abb. 3 b. Sanskrit-Texte können in Indien in verschiedenen Schriften geschrieben werden, in Devanagari, im Grantha-Alphabet oder in einer der lokalen Schriften der Südsprachen. In der Abbildung, die Paolino da S. Bartolomeo, Viaggio ..., Roma 1796, entnommen ist, werden Schriftzeichen des Devanagari den entsprechenden des Grantha-Alphabetes gegenübergestellt

\section{Sprachforschung im Dienst christlicher Missionsarbeit}

Wie sah es zu dieser Zeit auf andern Gebieten aus, beispielsweise in der Erforschung der indischen Religion, Philosophie oder Dichtung? Es war hier ähnlich wie in der Medizin! Ein genaues Textstudium war auch hier noch kaum möglich. Einige Theologen und Missionare sind jedoch hervorzuheben. Sie haben sich in besonderem Maße bemüht, in das einheimische Schrifttum, in die Religion und Philosophie Indiens einzudringen. Einer der ersten holländischen Prediger in Indien war D. Abraham Rogerius, der um 1630 das Evangelium an der Küste Koromandel lehrte. Zehn Jahre weilte er in Paliakatta, wo er zwei 
Brahmanen, Padmanaba und Damersa, gewinnen konnte, ihm die Geheimnisse der indischen Religion mitzuteilen. Für ihre Arbeit erhielt das Ensemble die finanzielle Unterstützung der Ostindischen Kompanie. Die Übertragung aus der indischen Sprache in das Holländische erfolgte ebenfalls wieder über das Portugiesische, welches Padmanaba beherrschte. Einen Teil seiner Ergebnisse hat Rogerius in dem 1651 in Leiden erschienenen Werk De Open-Deure tot het verborgen Heydendom veröffentlicht.

Den europäischen Geistlichen war die Erforschung der indischen Religion aber nicht so wichtig wie die Verkündigung ihrer eigenen. Im Laufe der Zeit bemühten sich zahlreiche Missionare, die einheimischen Sprachen zu erlernen, um durch sie den Indern das Evangelium zu lehren, christliche Moral zu predigen und Gebete sowie Lieder beizubringen. Der bekannteste Missionar der portugiesischen Zeit war der schon genannte Franziskus XaveRIUS. Er liegt heute in Goa begraben. Als großer Organisator der christlichen Mission erkannte er bald die Notwendigkeit, in Indien christliche Literatur in den einheimischen Sprachen vervielfältigen zu lassen. Wie wir schon hörten, übernahm diese Aufgabe sein Orden, die Societas Jesu. Am 6. September 1556 brachte man die erste Druckpresse nach Goa, und in den nächsten Jahrzehnten wurden Gebete und christliche Lehren in den indischen Sprachen Kunkani, Mahratti, Kannada, Tamil und andern gedruckt ${ }^{5}$.

Der Schwerpunkt der Sprachforschung der Missionare in Indien lag im allgemeinen natürlicherweise nicht so sehr auf der Ermittlung philologischer Details als mehr auf der Aneignung praktischer Worte und Sätze, die eine Verständigung mit den Indern ermöglichten. Und es hat wohl jeder Missionar im Laufe der Zeit einige Sprachkenntnisse erworben; doch es ist nur von manchen überliefert, daß sie die einheimischen Sprachen wirklich fließend beherrschten. Einer der bekanntesten katholischen Missionare in Indien war der italienische Jesuitenpater Roberto Nobili, der um 1600 nach Kotschin ging, um dort die für den Süden Indiens so wichtige Lingua malabarica (Tamil) zu erlernen. Später gründete er in Madure eine dank seiner guten Sprachkenntnisse erfolgreiche Missionsstation.

Um das Jahr 1660 kam es zum Zusammenbruch der Druckerei in Goa. Dieses Ereignis fiel in die Zeit zunehmender Depression der portugiesischen Macht in Indien. Die langen Fronten an den Küsten Afrikas, Persiens, Indiens, Ceylons, Hinterindiens und Indonesiens konnten einem feindlichen Angriff nicht gewachsen sein, wenn er an einer Stelle massiert erfolgte. Auf diese Weise kam es, daß vornehmlich die Holländer den Portugiesen eine Niederlassung nach der andern abnehmen konnten. Hand in Hand mit dem Rückgang der militärischen Macht ging auch der wirtschaftliche Zusammenbruch Goas einher. Und der dritte Geselle des Zusammenbruchs, die Seuchen, blieb auch nicht aus. Die Stadt wurde damals durch große Epidemien, deren Natur noch nicht ganz geklärt ist, heimgesucht. Die Bürger verteilten sich deshalb über die Orte der Umgebung oder reisten zurück nach Portugal. In die in starken Verfall übergehende Stadt zog schließlich noch die Malaria ein und verhinderte jeden systematischen Wiederaufbau.

Der Rückschlag, den die katholische Kirche durch den nachlassenden Einfluß ihrer Schutzmacht Portugal erhielt, war nur äußerlich. Die Missions-

${ }^{5}$ Der protestantische Prediger Philipp Baelde schrieb später, daß die Portugiesen «keine Kosten gescheut haben, um ganze Bücher in der malabarischen Sprache drucken zu lassen » (S. 189, in: Beschreibung ..., Amsterdam 1672). 
arbeit der Patres wurde trotz allem mutig fortgesetzt. In dieser Zeit reiste der zur Jesuitenmission Goa gehörende Pater Heinrich Rотн durch Zentralindien und studierte dabei Sanskrit und Hindi (20). Später fuhr er nach Rom und traf dort den nicht zuletzt wegen seiner naturwissenschaftlich-medizinischen Arbeiten bedeutenden Pater der Gesellschaft Jesu, Athanasius Kircher (1602-1680), der gerade sein Buch China illustrata vorbereitete. Roth brachte ihm hierzu fünt Tafeln «Hanscret» mit, vier Tafeln Elementa Linguae Hanscret und eine Tafel mit dem Pater noster und dem Ave Maria. Kircher nahm sie in sein Buch auf und ergänzte sie durch zehn weitere Tafeln mit mythologischen Bildern, die ebenfalls von Roth stammten und auf denen die Erklärung außer in Lateinisch auch in Hanscret gehalten war. Das Manuskript Kirchers konnte 1667 in Amsterdam gedruckt werden. Ein Holländer, W. VAN DER LAEGH, überarbeitete zuvor noch einmal die Tafeln und bereitete sie für das Setzen vor. Es ist interessant, das Asienwerk der Jesuiten gerade in Holland gedruckt zu finden, waren die protestantischen Holländer den Jesuiten doch besonders in indischen Dingen nicht gut gesonnen. Aber hier hat das koloniale Interesse an dem lehrreichen Buch wohl überwogen. Bemerkenswert ist ferner, daß Pater noster und Ave Maria nicht ins Sanskrit übertragen sind. Der lateinische Text ist lediglich mit indischen Schriftzeichen, in Devanagari, geschrieben.

Erforschung und Lernen des Sanskrit waren für die Missionare wichtig. Wurde diese Sprache auch kaum irgendwo von den Indern direkt gesprochen, so war sie doch die wissenschaftliche Sprache der Brahmanen, der geistig-religiösen Führer des heidnischen Indiens. Die Pandits, die gelehrten Brahmanen, waren die wirklichen Gegenspieler der christlichen Missionare. Mit ihnen hatte man sich letzthin auseinanderzusetzen; aber man konnte nicht einmal ihre Texte übersetzen, geschweige denn, wirklich mit ihnen diskutieren. Wie sollte man ihre Lehren widerlegen. War es den Missionaren auch häufig gelungen, in der städtischen, aus den verschiedensten Völkern und Volks- und Berufsgruppen gemischten Einwohnerschaft Übertritte zum Christentum zu erzielen, und konnten die Missionare hier besonders Angehörige der niedrigsten Kasten gewinnen, so gelang es doch nur schwer, einen Brahmanen zu bekehren. Es ist in diesem Zusammenhang nicht zu vergessen, daß selbst die Jesuiten lange brauchten, ehe jemand von ihnen philologisch detailliert ins Sanskrit eindrang. Hier ist vor allem Pater Johann Ernst Hanxleden aus Osnabrück zu nennen, der von 1700 bis 1732 in Malabar weilte (21). Er hat die erste bekanntgewordene Sanskrit-Grammatik zusammengestellt. Sie blieb jedoch nur Manuskript.

Ein anderer Pater, diesmal ein Karmeliter, der Österreicher Johann Philipp Wesdin, der in seinem Orden den Namen Paulinus a Sancto Bartolomaeo führte, brachte schließlich die erste Sanskrit-Grammatik zum Druck. Er war 1776 bis 1789 in Malabar und fuhr dann nach Rom, wo er in der indischen Bibliothek der Congregatio de Propaganda 
Fide $^{6}$ arbeitete und 1790 die Sidharubam seu Grammatica Samscrdamica veröffentlichte (22). 1802 erfuhr das Werk eine erweiterte Ausgabe.

In den Jahren 1674/75, etwa fünfzehn Jahre nachdem die portugiesische Druckerei in Goa ihre Arbeit eingestellt hatte, versuchten die Engländer, eine Druckerei in Indien zu errichten, und zwar in Bombay, das sie 1662 vom portugiesischen König als Geschenk erhalten hatten. Ziel dieser Druckerei war es, den Protestantismus zu propagieren (23). Inwieweit sie zu erfolgreicher Arbeit in den einheimischen Schriftsprachen gelangten, muß noch näher untersucht werden.

Aus der Zahl der protestantischen Prediger in Indien sei der Holländer Philipp BaLdaeus (BAELDE, geb. 1632) genannt, der seit 1656 in Indien wirkte. Er hinterließ uns das schöne Werk Beschrijving der Oost-Indische Kusten Malabar en Coromandel, benevens het Eiland Ceilon, das 1672 in Amsterdam erschien. Noch im gleichen Jahr kam am gleichen Ort auch eine deutsche Übersetzung dieses Werkes heraus. Baelde bringt in ihm ein Kapitel mit der Überschrift «Kurze Anleitung zur malabarischen Sprachkunst». Es handelt sich hier um Tamil. Baelde schreibt, noch nicht einmal die malabarischen Buchstaben seien bislang in einer niederländischen oder deutschen Presse gewesen. Man müsse diese Sprache lernen, wenn man die Heiden Südindiens bekehren wolle. Selbst Protestant, lobt er dann die gute Arbeit der Jesuiten auf diesem Sprachgebiet und hebt namentlich den Pater GasPar D'AQuilar hervor (24).

Baelde bediente sich, ähnlich wie Rheede van Drakenstein und andere Europäer, eines malabarischen Portugiesen als Dolmetscher. Er hieß Francisco und sprach außer der Lingua malabarica Portugiesisch. Baelde konnte ebenfalls Portugiesisch, und so verständigten sich beide über diese Sprache. Baelde schrieb christliche Texte portugiesisch auf, und Francisco übersetzte sie ins Malabarische. Ein anderer holländischer Prediger stand Baelde zur Seite, Joannes van Breyl. Auch er konnte portugiesisch sprechen. Wollten beide Missionare in den eroberten Städten verstanden werden, so mußten sie den protestantischen Glauben portugiesisch predigen. Es war dieser Zeit im ganzen orientalischen Raum von Sansibar bis Amboina nötig, zumindest Portugiesisch zu beherrschen, wenn man zu der einheimischen Bevölkerung näheren Kontakt gewinnen wollte ${ }^{7}$, da die Portugiesen in der «Romanisierung» der Küsten Asiens weit vorangekommen waren. Viele Menschen fühlten sich noch immer als Lusitano-Asiaten.

${ }^{6}$ Die Congregatio de Propaganda Fide hatte sich in indologischer Hinsicht damals schon einen Namen geschaffen, indem sie 1772 das Alphabeticum Grandonico-Malabaricum sive Samscrudonicum veröffentlichte. Von portugiesischen Missionaren war mit Unterstützung der Congregatio 1778 die Grammatica Marastta a mais vulgar que se practica nos reinos de Nizamaxu de Idalxa offericida aos padres Missionarios dos dittos reinos in ihrer Heimatsprache herausgebracht worden.

${ }^{7}$ Im Jahre 1698 wurde die Charta der Englisch-Ostindischen Gesellschaft erneuert. Damals forderte das Parlament den Einschub einer «missionary clause», nach welcher in jede Handelsniederlassung der Engländer in Indien ein «minister of religion » gesandt werden sollte. Dieser müsse Portugiesisch können, um überhaupt erst einmal bei den einheimischen Bediensteten der Europäer Gehör zu finden (Seyed Nurullah und J.P. NaIK, A History of Education in India during the British Period, 2. edition, Bombay/.../London 1951, S. 52). 
Baldaeus scheint nicht zu tief ins Tamil eingedrungen zu sein. In seinem Buch finden sich außer alphabetischen Lettern nur das Pater noster (im Gegensatz zu Kircher und Roth allerdings in die malabarische Sprache übersetzt) und ein Glaubensbekenntnis. Er wies darauf hin, daß die malabarische Sprache recht schwer sei. Zwar hat er vermerkt, daß er eine vollständige Grammatik mit sich führe; er sagt aber nirgendwo, daß er selbst das Malabarische erlernt hat. Gebete und Grammatik dürften also hauptsächlich aus portugiesischen Vorarbeiten stammen oder von Dolmetschern erarbeitet sein. In seiner Beschreibung von Malabar, Koromandel und Ceylon finden sich hin und wieder medizinische Einzelheiten eingestreut. Soweit sie die indische Medizin betreffen und nicht aus älteren Beschreibungen übernommen sind, wird er sie wohl ebenfalls meist in portugiesischer Sprache erfahren haben.

Unter den protestantischen Missionaren wird einer heute besonders verehrt. Es ist Bartholomaeus Ziegenbalg (1683-1719), ein Deutscher aus der Lausitz, der in dänische Dienste getreten war. Er nimmt bei den Protestanten etwa den Platz ein, den in der katholischen Kirche Franziskus Xaverius innehatte. Ziegenbalg kam 1706 nach Trankebar, einer dänischen Station in Indien. Vermittels eines Dolmetschers nahm er den Kontakt zur einheimischen Bevölkerung auf, lernte aber bald selbst Portugiesisch und Malabarisch (Lingua malabarica $=$ Tamil). Innerhalb weniger Jahre soll er das Malabarische so gut beherrscht haben, daß er darin predigen und Christenlehre abhalten konnte. Auch er erkannte schnell die Bedeutung von religiösem Schrifttum in der einheimischen Sprache für die Intensivierung der Missionsarbeit. So wurde 1712/13 unter seiner Leitung in Trankebar eine,portugiesische und malabarische Druckerei errichtet. Die drei Setzer waren Deutsche. Von Ziegenbalgs indologischen Arbeiten seien hier die in Halle 1716 herausgekommene Grammatica Damulica sive Malabarica und eine Biblia Damulica genannt. Die Bibel wurde in den Jahren 1723 bis 1727 in Trankebar gedruckt.

Sein erfolgreiches Wirken fand nicht die volle Zustimmung des Kommandanten von Trankebar und der meisten dort wohnenden Europäer. Während die Portugiesen sogleich nach ihrer Ankunft eine Politik der Vermischung mit den Indern und die Portugisierung möglichst aller Leute betrieben hatten, war es das Bestreben der Engländer und Holländer sowie auch der Dänen, möglichst große Distanz zu der einheimischen Bevölkerung zu halten, sie nicht zu europäisieren, sie nicht gleichberechtigt werden zu lassen usw. Man fürchtete, der Europäer würde nach Erziehung, Ausbildung und Christianisierung der Eingeborenen seine Vormachtstellung verlieren. Außerdem hörten die im Orient ein lockeres Leben führenden Kolonialherren nicht allzugern moralische Mahnworte. So soll es schließlich soweit gekommen sein, daß der norwegisch-deutsche Kommandant Hassius die protestantischen Missionare habe prügeln und Ziegenbalg als Aufrührer ins Gefängnis werfen lassen. Um die selbst hier noch gefürchtete Feder des letzteren auszuschalten, wurden ihm ausdrücklich Tinte und Papier verweigert. Später kam es zur Abberufung des Kommandanten, und die Arbeit der Missionare fand in steigendem Maße Zustimmung; ja gerade in den dänischen Niederlassungen wurde die Mission fortan besonders aktiv betrieben.

Im Kreis der philologisch interessierten Mitarbeiter von Ziegenbalg war auch einer, der ein medizinisches Thema in Angriff nahm, JoHAnnes Ernst GRÜNDLER (gest. 1728). Die Missionare schrieben aus Trankebar zahlreiche Briefe nach Europa, in denen sie über ihre Arbeit und das, was sie in Indien 
an Besonderem fanden, berichteten. Ein Brief vom 3. Oktober 1709 enthält die Notiz, daß ein malabarischer Arzt Ziegenbalg einen langen Katalog gezeigt habe, in dem zahlreiche "Species morborum » aufgeführt seien, sowie ein Lexikon mit den Namen von Krankheiten (26). Am 10. September 1712 berichteten Ziegenbalg und Gründler in einem Brief an Professor August Hermann Francke in Halle, daß sie medizinische, in der malabarischen Sprache (Tamil) geschriebene Bücher gesammelt und daraus einen Traktat über die Arzneikunst der Heiden zusammengestellt hätten. Dieses sei auf Begehren einiger Ärzte in Europa geschehen (27). In einem Brief vom 22. Oktober 1712 schreiben Ziegenbalg und Gründler, daß sie vier deutsche Traktätlein in einem Paket an Herrn Böhme abgesandt haben, unter denen ein medizinisches aus den Büchern der heidnischen Ärzte in Trankebar kolligiertes Traktat sei (28).

Wir erfahren aus einem andern nach Kopenhagen gesandten Brief (Trankebar, 17. September 1712) den Titel (29) des damals angekündigten Traktates: Der malabarische Medicus, welcher kurzen Bericht gibt, teils was diese Heiden in der Medizin für Prinzipien haben, teils auf was für Art und mit welchen Medikamenten sie die Krankheiten kurieren, den Herrn Medicis in Europa zu dienlicher Nachricht und Prüfung aus den medizinischen Büchern dieser Malabaren zusammengetragen und übersetzt von J.E.G., nebst einer Vorrede, darinnen eine Sciagraphia medica eines Brahmanen miteingeführt ist, in welcher er die Ordnung zeigt, wie ihre Medici das ganze Studium medicum in ihren Schulen treiben.

Am 9. September des Jahres 1713 wiederholen Ziegenbalg und Gründler in einem Brief an Professor Francke die Mitteilung, daß ein medizinisches Traktat mit englischen Schiffen nach England gesandt sei und von dort nach Deutschland gelangen sollte, und fragen, ob das Traktat richtig eingetroffen sei. (30). Es gelangte auch tatsächlich nach Halle und ist dort noch heute in der Bibliothek erhalten, nach einer Mitteilung von deren Leiter, Jürgen Storz, allerdings in einem in Zerfall begriffenen Zustand.

Wichtige Teile dieses Traktates sind 1727 in Nürnberg in der Appendix ad Actorum Physico-Medicorum Academiae Caesareae Leopoldino-Carolinae Naturae Curiosorum in Germania und an andern Stellen auch tatsächlich veröffentlicht worden. Bei diesem medizinischen Traktat handelt es sich also nur um eine «Kollektion» aus malabarischen Büchern, die wohl vorwiegend in Tamil geschrieben waren. Das Werk Medicus Malabaricus ist also nicht eine Edierung und Wort-für-Wort-Übersetzung eines einzigen Textes, sondern eine Auslese leicht zu verstehender und unter Mitarbeit 
einheimischer Ärzte übersetzbarer Einzelheiten, die von Gründler zu einer neuen Abhandlung zusammengestellt worden sind. Daß Gründler allerdings selbst relativ gute Kenntnisse des Tamil besessen haben muß, geht schon aus seiner Mitarbeit bei Ziegenbalgs umfangreichen grammatikalischen Arbeiten und Bibelübersetzungen hervor. Wir dürfen Gründler also als ersten Europäer ansehen, der einzelne medizinische Kapitel aus indischen Büchern übersetzt und Proben daraus in Europa zum Druck gebracht hat!

Die Neugier der Ärzte, die von der Übersetzung Gründlers hörten, war zunächst recht groß; allein nach dem Bekanntwerden des Werkes blieb das Echo gering. Eigentlich fand das Werk Gründlers nur bei einem Medizinhistoriker, bei Heinrich Schulz, näheres Interesse. Er referiert in seiner 1728 in Leipzig erschienenen Historia medicinae einige ihm wichtig erscheinende Einzelheiten. Schulz kam zu dem Schluß, daß die Inder zwar zahlreiche Theorien hätten, daß aber viele davon offensichtlich falsch seien. Die Prinzipien der indischen Medizin seien wahrscheinlich so alt wie die der alten Ägypter. Vor allem hob Schulz ein indisches Werk hervor, das Wagadasastirum (= die sechs Bücher der Medizin). Darin vermisse man die anatomisch-physiologischen Entdeckungen, so z.B. den Blutkreislauf. Die Inder hätten schlechte anatomische Kenntnisse, wenn 72000 Arterien ihren Ursprung vier Finger breit unterhalb des Nabels hätten und sich danach im ganzen Körper verteilten. Ihre Vorstellungen über die Entstehung des Pulses unterhalb des Nabels seien falsch, und genauso unbrauchbar seien ihre Theorien über die Atmung. Mit Erstaunen wird Schulz gelesen haben, daß die Inder die große Zahl von immerhin 792 Geisteskrankheiten hätten und daß es nach ihrer Ansicht insgesamt 2847 Krankheitsarten gebe ${ }^{8}$.

${ }^{8}$ In Ergänzung zu Gründler und Schulz sei der Engländer EduARD Ives zitiert. Er kam 1754 als Wundarzt nach Südindien, vornehmlich an die Küste Koromandel, wo sich zu dieser Zeit England und Frankreich feindlich gegenüberstanden. Ives achtete auch auf die einheimische Medizin und besuchte deshalb mehrere portugiesische «schwarzhäutige» Ärzte, so einen namens Diego. Von ihm schrieb Ives: «Er zeigte uns einen artigen Vorrat von Arzneiwaren, unter denen wir einige sehr schätzbare Sachen fanden.»

Während der Engländer viel von den praktischen Kenntnissen der malabarischen Ärzte hielt, fand ihre Literatur seine Geringschätzung. An einer andern Stelle seines 1773 in London erschienenen Buches $A$ Voyage from England to India heißt es nämlich: «Die Wissenschaft der indischen Ärzte ist sehr eingeschränkt. Ich und Herr Thomas verlangten einmal von einem dieser schwarzen Ärzte, er solle uns ein Verzeichnis der Arzneien aufsetzen, die sie gewöhnlich gebrauchten. Anstatt eines solchen Verzeichnisses brachte er uns eine Menge Blätter, Pflanzen, Rinden und Wurzeln von verschiedenen Bäumen, die 
Durch die vielfältigen Unternehmungen war Trankebar in der protestantischen Welt bald wohlbekannt. Doch später wurde es durch eine andere Stadt überflügelt. Die Dänen verlagerten nämlich den Hauptsitz ihrer Missionsarbeit nach Frederiksnagar im reichen Bengalen. Die Stadt war seit 1676 dänisch. Sie wurde meist Serampore genannt. Hier gründeten die Serampore-Missionare (Baptisten) mit Unterstützung der Regierung Schulen, eine Druckerei und eine Papierfabrik, und es wurden in Zusammenarbeit mit englischen Missionaren verschiedene Wörterbücher und Grammatiken verfaßt sowie religiöse und wissenschaftliche Bücher in Bengali, Telugu, Mahratti und Sanskrit übersetzt. Umgekehrt kam es auch zur Übersetzung aus dem Sanskrit; so wurde z. B. das Epos Ramajana übertragen. Im Jahre 1818 gründeten die Dänen schließlich in Serampore ein philosophischtheologisches College, das auch kurz Universität genannt wurde. Serampore blieb bis 1845 dänisch; dann ging es an die Engländer über.

\section{Sprachforschung unter der englischen Herrschaft über große Gebiete Indiens}

Im Jahre 1756, während Frankreich und England sich in Südindien feindlich gegenüberstanden, eroberte der Nabob von Bengalen, Suradschud-Daulah, das Fort William (Kalkutta). Die dort gefangenen Engländer wurden unmenschlich behandelt. Nun ging General Roвert Clive (1725-1774) zum Gegenangriff über. Er eroberte Kalkutta zurück und vernichtete 1757 bei Plassey das weit überlegene Heer des Nabobs. Von jetzt ab gehörte England die Vorherrschaft im Nordosten Indiens, und es erwarb nach und nach immer neue Territorien.

Der Sieg von Plassey ist darum so bedeutungsvoll, weil es dadurch zum erstenmal einer europäischen Macht gelang, weite Landstriche auch im Innern von Indien in die Hand zu bekommen. Bisher hatte sich die Herrschaft der Europäer - der Portugiesen, Holländer, Dänen, Franzosen und Engländer in gleicher Weise - nur auf Küstenstriche erstreckt. Hier hatten sie Handelsniederlassungen und Festungen angelegt. Über zweihundert Jahre hatten die «Portugiesen» an den Küsten eine führende Rolle inne, auch noch unter fremden, nordwesteuropäischen Besatzungen. Sie waren das Bindeglied zwischen den Indern und den Vertretern der einzelnen europäischen Ostindien-Gesellschaften. Wie schon gesagt, fiel ihnen die wichtige Funktion des Dolmetschens zu. In den Kloster- und Missionsschulen

sie, wie er sagte, gemeiniglich abkochten. In Fiebern gebrauchten sie Pfeffer und andere erhitzende Dinge. Wir fragten ihn, ob sie gar keine geschriebenen Aufsätze von ihrer Methode und Art zu verfahren hätten? Er antwortete: ja, und brachte uns ein großes Buch, welches aus Blättern vom Palmbaum (Phoenix dactylifera) bestand, auf welche ihr Bericht aufgeschrieben war. Wir ließen uns einen Teil dieses Buches durch einen Conucopola übersetzen. Es war in dem schwülstigen orientalischen Stil geschrieben, fing mit großem Preis des Allmächtigen für die wunderbare Bildung der Menschen an; ging hierauf zu der Bemerkung über, daß der Mensch aus zweimal hunderttausend Teilen bestünde, zehntausend wären aus Adern gemacht, zehntausend aus den Nerven, siebzehntausend aus Blut, eine gewisse Zahl aus Knochen, Lymphe usw. Alles dieses war ohne Ordnung und Plan niedergeschrieben. Dieser Art, fanden wir, waren alle ihre geschriebenen Aufsätze über die Arzneikunst» (E. Ives, Reisen nach Indien und Persien, aus dem Englischen von Christian Wilhelm Dohm, Leipzig 1774, S. 80 und 95). 
hatten sie ihre Ausbildung erhalten und taten Dienst in den Büros der Faktoreien. Die Portugiesen vermittelten die Waren und knüpften den Handel zwischen Faktorei und Indern. Sie dienten auch in den Streitkräften der Ostindien-Gesellschaften und wurden dort nicht nur als Dolmetscher, sondern auch als tapfere Soldaten gelobt.

Die Portugiesen in den holländischen, englischen, dänischen und französischen Niederlassungen waren aber nach 1600 meist keine reinrassigen Leute, sondern braunhäutige Mestizen. Die Südinder nannten sie Duibasch oder Topas, was soviel wie Mestize oder Mischling, Mann mit doppelten Qualitäten oder Dolmetscher heißt(31). Die Topasi hatten ihre Bedeutung aber nur an den Küsten; im Innern des Landes besaßen sie keinen nennenswerten Einfluß; ihre Zahl reichte für die großen Territorien nicht aus. Hier fehlten auch die portugiesischen Elementarschulen, aus denen die Topasi, die Bediensteten der Nordwesteuropäer in Indien, bildungsmäßig hervorgingen. Die Engländer waren infolgedessen in ihren neuen Gebieten darauf angewiesen, Angestellte und Bedienstete allein aus der indischen Bevölkerung, das heißt Mohammedaner und Hindus, zu nehmen.

Aus dieser Situation resultierte, daß sich die Engländer von nun an entschieden aktiver als bisher für das einheimische Schulsystem und die Ausbildung der Leute, mit denen sie die Verwaltung der Kolonialgebiete unterhalten wollten, interessierten. Man dachte vor allem an eine Zusammenarbeit mit den vornehmen Kasten, zumal sich diese damals mehr zu den Europäern als zu den Angehörigen der niederen Kasten hingezogen fühlten. Die Söhne der Vornehmen sollten in Sprache, Geographie, Geschichte, Mathematik und Naturwissenschaften ausgebildet werden. Die Engländer glaubten, den Wünschen der Einheimischen sogar besonders entgegenzukommen, wenn sie deren alte Kultur erhielten, sie an Colleges mit Hochschulcharakter pflegten. So wurde 1780 durch den Generalgouverneur Warren Hastings in Kalkutta ein mohammedanisches College (Medrese) und elf Jahre später, 1791, zu Benares durch den dortigen Residenten Jonathan Duncan ein Sanskrit-College für die Söhne der Brahmanen und Väidjas ins Leben gerufen.

Im Laufe der Jahrzehnte unternahmen die Engländer, Moslems und Hindus die verschiedensten Versuche, zu einer endgültigen und für alle Teile günstigen Lösung in der Frage der Ausbildung der Jugend zu kommen. Sollte die Erhaltung von Kultur und Tradition der Inder im Vordergrund der Erziehungsarbeit stehen oder sollte die Jugend zum Fortschritt und zum leichteren Gebrauch der europäischen Zivilisationsgüter erzogen werden? Sollten sich die Inder in Zukunft besser in den indischen oder in den europäischen Dingen auskennen, usw.?

Das Ergebnis der Ausbildung an den indischen Colleges war für die Verwaltung der englischen Kompanie unbefriedigend, da die Studenten zu sehr in Richtung Philologie und alte Literatur ausgebildet wurden, und so 
gründeten die Engländer um 1800 ein weiteres College, das Royal College im Fort William zu Kalkutta. Es wurde auch einfach Universität genannt. Hier sollten die zukünftigen höheren Angestellten der englischen Administration in den einheimischen Sprachen und kulturellen Dingen, in Mathematik und Naturwissenschaft unterrichtet werden. Auch Engländer studierten hier. Die Engländer wollten ihre Leute so ausbilden, daß sie sich selbst und ohne die Vermittlung geschworener Dolmetscher ein Bild von den indischen Angelegenheiten machen konnten. Es wurden verschiedene Sprachen, neben Englisch, Französisch und Portugiesisch, Bengali, Hindi und Persisch, Tamil und Telugu gelehrt (32).

Ein College in Kalkutta, das Universitätscharakter hatte, schien manchen Engländern aber doch zu gefährlich. Inder mit den gleichen Kenntnissen wie die Europäer würden sich auf die Dauer nicht regieren lassen! Das College wurde deshalb schon nach kaum zwei Jahren, am 27.Januar 1802, durch den Court of Directors der Ostindischen Kompanie wieder aufgehoben. Statt des Colleges in Indien gründete man jetzt ein Oriental College in London. Hier wurden englische «Schreiber» für Indien ausgebildet. Man glaubte, auf diese Weise die Entwicklung in dem aufblühenden Indien besser in der Hand zu behalten.

Im Laufe der Jahrzehnte entstanden an den verschiedensten, unter britische Hoheit gelangten Orten weitere Sprach-Colleges. Sie glichen im wesentlichen dem Stil der Colleges von 1780 und 1791. Viele Inder standen den Sprach-Colleges, besonders den Sanskrit-Colleges, abweisend gegenüber. Sie hielten ihre Gründung für einen Stillstand oder gar für einen Rückschritt in der Entwicklung; denn man würde dort Sprachen erlernen, die für den Fortschritt des Landes kaum noch einen praktischen Nutzen hätten. Diese Haltung geht auch aus einem Memorandum hervor, das der Radscha Ram Монan Roy, ein Pionier der Sozialreform und «English education» in Indien (33), anläßlich der 1824 bevorstehenden Eröffnung eines SanskritColleges in Kalkutta absandte. Darin hieß es: Die Regierung solle nicht das streng gehandhabte Sanskrit-College zu Kalkutta fördern, sondern eine mehr liberale Einrichtung schaffen, in der Mathematik, Naturphilosophie, Chemie, Anatomie und andere «useful sciences» gelehrt werden. Man sollte talentierte Leute zur Ausbildung nach Europa senden und sie nachher in Indien unterrichten lassen. Ein solches neuartiges College sollte mit Büchern, Instrumenten und Apparaten versehen werden. Der Radscha schlug schließlich noch Englisch als alleinige Sprache für die Naturwissenschaften vor (34). 
Bleiben wir bei der Entwicklung in Bengalen, in Kalkutta, da sie in Indien führend war. Die Regierung konnte auf die Dauer dem drängenden Wunsch der Mehrheit der vornehmen Jugend, praktisch anwendbare Naturwissenschaften und westliche Medizin zu studieren, nicht ausweichen. So suchte sie einen Kompromiß zwischen den verschiedensten konservativ eingestellten und den fortschrittlichen Richtungen sowie mit den englischen philanthropischen Kreisen. Im Jahre 1826 wurde je eine «Medical class» an das Calcutta Sanskrit College und an die Calcutta Medrese (TibbiMedrese $=$ Medizinschule) angeschlossen. Neben das Studium der alten Texte der Hindu-Medizin oder der mohammedanischen Junani-Tibb (ionischarabisch-persische Medizin) trat jetzt ein Unterricht in Anatomie, Chirurgie und Medizin, aber vorerst nur an Hand von englischen Büchern (35). Die ärztliche Praxis erlernte der Student dann bei einem Väidja oder Hakiem oder als Gehilfe in einem europäisch geleiteten Spital. Die nicht an westliche Bücher gebundene praktische Ausbildung geschah also noch 1826 in der gleichen Weise wie schon lange Zeit vorher. Übrigens hatten die indischen Ärzte schon vor 1826 die Möglichkeit, «Native Doctor» zu werden. Zu diesem Zweck gingen sie in die schon erwähnten europäischen Hospitäler und arbeiteten hier mehrere Jahre, bis man ihnen die Bezeichnung «Native Doctor» zuerkannte. Es war dies eine rein praktische Ausbildung.

Ein erster Versuch, die Native Doctors auch theoretisch in systematischer Weise zu unterrichten, wurde bereits vier Jahre vor der Lösung von 1826 unternommen. Im Jahre 1822 gründeten die Engländer in Kalkutta die «Native Medical Institution for Training Native Doctors». Diese Schule wurde unter die Leitung eines Medical Officers gestellt. An ihr lehrte man auch Anatomie und Pathologie. Da damals die Inder sich aus magischreligiösen Gründen noch weigerten zu sezieren, Anschauungsunterricht aber billigten, gingen die Studenten in das General Hospital, wo man bei den Sektionen von Europäern zusah und die indischen Toten äußerlich und nur palpierend betrachtete.

Endlich, 1835, wurde in Kalkutta ein Medical College eröffnet. Es war eine medizinische Akademie, in der Ärzte nach westlichem Vorbild in englischer Sprache ausgebildet wurden. Die Bahn war frei für die Europäisierung der indischen Medizin. In den nächsten 125 Jahren erfolgte die Gründung zahlreicher weiterer Medical Colleges, und es wurden nach und nach auch zahlreiche Universitäten nach westlichen Vorbildern ins Leben gerufen. 


\section{Madhusudana Gupta}

Viele junge, auf Fortschritt bedachte Inder strebten seit Anfang des vorigen Jahrhunderts nach einer europäischen Ausbildung. Wie stand es mit ihren indischen Sprachstudien? Beschränken wir uns auf die Mediziner. Nur ganz wenige Studenten des Medical College von Kalkutta beschäftigten sich auch noch mit der alten einheimischen Literatur. Zu ihrer Fortbildung wurden im Laufe der Jahre am College zwar verschiedene Sprachklassen eingerichtet; diese dienten aber weniger dem Studium der alten einheimischen Literatur als vielmehr der Erlernung von Sprachen, in denen man die Patienten nach ihren Beschwerden befragen konnte.

Ein Mann des Medical College machte in der Ausbildung der Mediziner eine Ausnahme. Er hieß Madhusudana Gupta. Sein Name wurde dadurch bekannt, daß er als erster Inder eine Leiche sezierte - ein Unternehmen, das bislang als Vergehen galt. Madhusudana Gupta wurde dann auch Lecturer of Anatomy am Medical College zu Kalkutta. Er ist gleichzeitig der Herausgeber der ersten gedruckten Ausgabe des alten medizinischen SanskritWerkes Suschruta. Es erschien in den Jahren 1835/36 in Kalkutta.

\section{Medizinische Schriftstellerei in Indien nach 1498}

Heute werden in Deutschland unter dem Begriff «Indische Medizin » fast nur noch die Werke von Suschruta, Tscharaka und Vagbhata sowie das 1890 von Leutnant Bower (36) in Ostturkestan entdeckte Manuskript verstanden. Es erhebt sich die Frage, ob die Inder in den vergangenen Jahrhunderten nicht noch andere Werke hatten, vor allem, ob sie nach 1498, nachdem sie durch die Portugiesen Kenntnis von der europäischen Medizin erhielten, keine eigenen Werke mehr verfaßt haben. Es wurde schon darauf hingewiesen, daß die Inder tatsächlich noch andere Manuskripte besaßen als nur die großen vier. Wenn wir Europäer in Anbetracht dieser Manuskripte in dreihundert Jahren aber dennoch keine U̇bersetzung eines längeren medizinischen Textes zustande brachten, so läßt sich fragen, ob das vielleicht daran lag, daß Indien nichts Neues bot, was einer solchen Arbeit wert war? Und nach den von mir bereits kurz zitierten, vor 1800 erfolgten Übersetzungen von Vokabeln aus Wörterbüchern und den Übersetzungen kleinerer Texte, vor allem aber nach dem negativen Echo, das die Übersetzung Gründlers gefunden hat, könnte man wirklich der Ansicht sein, die medizinischen Bücher Indiens hätten außer einigen Namen von Krankheiten sowie der Beschreibung von Medizinaldrogen und ihrer Anwendung keine 
Dinge enthalten, die für die europäische Pathologie und Therapie von Interesse waren. Die vor 1800 bereits bekanntgewordenen größeren Werke hielt man für längst veraltete Werke aus der klassischen Zeit Indiens, beziehungsweise für ebenfalls schon veraltete Glossare, die eigentlich nur noch philologisch-medizinhistorischen Wert hätten!

Inzwischen haben die Bibliothekskataloge reichlich gezeigt, daß es in Indien bis etwa 1800 tatsächlich eine fleißige und sogar eigenständige medizinische Schriftstellerei gab, zum Teil in Sanskrit, zum größeren Teil aber in Persisch sowie in mehreren anderen Landessprachen vom Urdu bis zum Singhalesischen. Sie beschränkte sich keineswegs nur auf Kompendien von Heilpfianzen und deren Wirkungen. Winternitz hat schon 1920 auf einige Sanskrit-Monographien über spezielle Gebiete der Medizin hingewiesen (37); so nannte er beispielsweise Fachbücher für Fieber- und Kinderkrankheiten, über Augenleiden, Pulsfühlen usw. Er erwähnte Kajasta Kamunda, der 1489 ein Buch über Fieber und dessen Behandlung veröffentlicht hat. Es heißt Jvaratimirabhaschkara. Selbst nach 1498 wurden noch zahlreiche Monographien verfaßt. So hat im 16.Jahrhundert Bhavamischra das Werk Bhavaprakascha geschrieben. In ihm wird die von den Europäern eingeschleppte Syphilis erwähnt, wodurch es allein schon nach 1498 datiert werden kann. Im 17.Jahrhundert schrieb der Dichter Lolimbaradscha ein Lehrbuch der Therapie in Versen, das Väidjadschivana.

In der Zeit zwischen 1500 und 1750 wurden die schöpferischen Literaturstudien in Indien vor allem an den Höfen der Könige und Fürsten betrieben. Mehrere dieser Mäzene erwarben sich dadurch einen guten Ruf. Sie versammelten außer Dichtern, Schriftgelehrten, Historikern, Mathematikern und Astronomen auch Ärzte sowie Dolmetscher um sich. Besonders interessiert an der indischen Medizin waren die Mohammedaner, sowohl reisende arabisch-persische Ärzte wie auch die neuen Landesfürsten, so der in Nordwestindien regierende türkisch-afghanische Schah SiKander. Unter ihm kam es zu einem festen Zusammenfluß verschiedener Sprachen zu einer neuen, dem heute in Pakistan vorherrschenden Urdu. Dieses setzt sich aus arabischen und persischen Elementen und aus Worten des Hindi, Gudscheratit und Sindi zusammen. Sikander ist allerdings nicht gerade ein Mäzen gewesen. Er war ein fanatischer Anhänger des Islams und schuf sich durch die Zerstörung von Tempeln, Schulen und Bibliotheken der Hindus und Buddhisten einen traurigen Ruf. Anderseits war er bestrebt, aus den Schriften der Inder, und da besonders aus den medizinischen, wertvoll erscheinende Einzelheiten herausziehen zu lassen. Er ordnete deshalb die Kompi- 
lation und Übersetzung medizinischer Werke an und rief in Agra gelehrte indische Ärzte zusammen, ergänzte sie durch Ärzte aus Arabien und Persien und selbst aus Kasachstan, der Heimat des weitbekannten und hochangesehenen Arztes und Schriftstellers Avicenna. Sie schufen gemeinsam das große Kompendium der Medizin, das den Namen Sikanders erhielt: TibbiSikandari (= Medizin Sikanders) (38).

Im Dekkan regierte zwischen 1518 und 1687 die Kutub-Schah-Dynastie. Um das Jahr 1590 entschloß sich Moнammed-Kuli-Kutub-Sснан, eine neue Hauptstadt zu errichten; es entstand Haiderabad, das bald zu einer führenden Stadt der Künste und Bildungsstätten wurde. Mehrere Medresen, das sind höhere Schulen für Grammat k und Theologie, dienten der Fortbildung der Jugend. Im Jahre 1595 wurde dort auch ein Hospital (Dar-usSchifa) gebaut. Ihm war eine Medizinschule für Hakiems eingefügt. Hier wie auch sonst in Haiderabad versammelten sich Ärzte, Hakiems und Väidjas zu wissenschaftlichen Diskussionen. Sie fanden die Förderung der Dynasten, wenn sie sich als medizinische Schriftsteller betätigten (39).

Es ist in diesem Aufsatz nicht möglich, alle Literaturzentren Indiens aufzuzählen. Eines sei jedoch genannt: Benares. Uns interessiert dort besonders ein französischer Arzt aus Montpellier, FrançoIs Bernier (geb. ? - gest. 1688) und sein Bericht über Literaturstudien in der heiligen Stadt Benares am heiligen Ganges. Es handelt sich um die wichtigste Bildungsstadt der Brahmanen. Diese hatten in alter Zeit noch keine geschlossene Universität, Medrese oder Akademie mit Klassen, sondern der Unterricht erfolgte vornehmlich in den Gärten der Vorstädte. Dort saß der Lehrer, und um ihn herum versammelten sich die Schüler. Auf einen Lehrer kamen dabei zwei bis höchstens fünfzehn Schüler. Rund zwölf Jahre weilte der Lernende bei den Pandits von Benares. Mäzene, Fürsten und reiche Kaufleute unterstützten Lehrer wie Lernende ${ }^{9}$.

Um das Jahr 1665 hatte der Gouverneur von Benares, Dara Scheko, der älteste Sohn des Großmoguls Sснан Jehan, in seiner Residenz einen.

9 Noch 150 Jahre nach Bernier fand in Benares der Unterricht in der gleichen Weise statt. Walter Hamilton gibt davon einen anschaulichen Bericht. Er war 1801 in Benares. Damals existierte zwar schon das von Jonathan Duncan gegründete "Public College of Hindu Literature"; außer diesem gab es aber in der Stadt etwa 300 Privatlehrer allein schon für das Hindurecht. Sie hatten etwa 5000 Schüler. Damit kamen auf jeden Lehrer etwa 16 Schüler, also genau so viele wie zur Zeit von Bernier. Hamilton vermerkte weiter, daß Lehrer und Schüler noch 1801 von reichen Pilgern, Hindu-Prinzen und MahrattenFürsten unterstützt wurden (A Description of Hindoostan, Band 1, London 1820, S. 307). 
Stab von Gelehrten versammelt. Pandits erklärten beispielsweise die Upanischaden, das sind indische Lehrkapitel über die Natur, Gott und den Menschen. Dolmetscher übertrugen den Text der Pandits ins Persische. Doch man interessierte sich im Mogulreich nicht nur für die indische Kultur, sondern auch für die europäische. Brennend wollte man Neues über Europa erfahren. So ließen sich Kaiser, Könige und Fürsten von Missionaren und Reisenden Gemälde und Kupferstiche schenken. Kleine Bilderalben waren besonders gefragt. Sie vermittelten einen Eindruck von der europäischen Welt, die damals kaum ein Inder reisend zu Gesicht bekam. Dem Europäer gehörte deshalb allein schon aus dieser Neugier heraus immer wieder die Gastfreundschaft der Inder. In Benares fand dann auch Bernier bei Dara Scheko eine Anstellung. Er wurde vom Gouverneur damit beauftragt, die modernste medizinisch-naturwissenschaftliche Literatur Europas mit Hilfe von Dolmetschern ins Persische zu übersetzen. Persisch war damals die führende Schriftsprache im islamisch beherrschten Indien. So ist es nicht zu verwundern, daß an den Fürstenhöfen immer wieder Persisch-Dolmetscher gefunden wurden, die zu den verschiedenen indischen Sprachen vermittelten, sich aber auch mit der Übertragung von Texten ins Persische beschäftigten. Doch zurück zu Bernier. Er ließ zunächst William Harvey ins Persische übersetzen, da die Lehre vom Blutkreislauf höchst aktuell war. Ferner wurde die Arbeit von Dr. Jean Pecquet (1622-1674), einem Studienfreund Berniers aus Montpellier, übertragen, in welcher die Entdeckung der Chyluszisterne und des Milchbrustganges beschrieben wird. Fünf Jahre lang war Bernier mit derartigen Übersetzungen beschäftigt.

Bernier erfuhr seinerseits in Benares auch einiges aus indischen Werken, die in Versen geschrieben waren. Wir hören bei Bernier leider nirgends, ob er selbst indische Werke übersetzen konnte. Er staunte über das Wissen der Pandits, das recht umfangreich sei; sogar die Atomtheorie des DemoKRIT und EPIKur sei ihnen bekannt. Die Pandits waren ihrerseits begierig, von Bernier die erst neuerdings in Europa vertretenen Thesen über die kleinsten Teilchen der Materie zu erfahren. Er muß ihnen also auch die Lehre der namhaften Atomisten der Neuzeit, vor allem die des Pariser katholischen Priesters Pierre Gassendi (1592-1655) zur Übersetzung mitgeteilt haben. Ferner arbeitete man über Descartes (40). Ob Manuskripte der Übersetzungen von Bernier erhalten sind, weiß ich nicht. Da es sich in Indien zu dieser Zeit nicht um gedruckte Bücher, sondern um Handschriften handelte, deren Verfielfältigung durch Abschreiben erfolgte, dürften überhaupt nur wenige Exemplare entstanden sein! 


\section{Erste Blütezeit europäischer Sanskrit-Philologie}

Ein Inder, Madhusudana Gupta, war es, der den Europäern das erste große medizinische Werk seiner Heimat in Form eines zweibändigen Buches präsentierte. Gab es denn überhaupt keine Europäer, die so weit in die indische Literatur eingedrungen waren, daß sie einen langen Text Wort für Wort übersetzen konnten?

In der zweiten Hälfte des 18. Jahrhunderts begannen verschiedene Europäer, indische Texte systematisch zu sammeln und einzelne auch schon zu übersetzen. Hier sei der Orientalist Abraham Hyacinthe Anquetil du Perron (1731-1805) genannt, der 1755 in Kalkutta landete und verschiedene Städte aufsuchte, um Handschriften aufzutreiben und die entsprechenden Sprachen zu erlernen. Vor allem hatten es ihm die Schriften der Parsen angetan. Zu ihrem Studium weilte er längere Zeit in Surat, der mehrere hunderttausend Menschen zählenden Großstadt an der Westküste Indiens. Nach seiner Rückkehr nach Paris konnte er 1762 der Bibliothèque du Roi rund 180 Manuskripte übergeben. Anquetil du Perron widmete seine Aufmerksamkeit auch dem Sanskrit, hat es jedoch nicht perfekt erlernt (41).

Während der Franzose Anquetil du Perron als reiner Wissenschaftler fast immer mit finanziellen Schwierigkeiten zu kämpfen hatte und schließlich auch völlig verarmt starb, haben es einige Engländer relativ besser gehabt. Sie dienten im Civil Service der East India Company und hatten so neben ihrem Dienst Gelegenheit, an Ort und Stelle auch die einheimischen Sprachen und Sitten und vor allem das Sanskrit zu erlernen. Da die Engländer sich in den neuerworbenen, großen Landstrichen mit der einheimischen Bevölkerung auf rechtlicher Basis auseinandersetzen mußten, wenn sie die Ordnung im Lande erhalten wollten, so begrüßte die Company es, wenn sich jemand von den ihr dienenden Engländern auch sprachlich mit dem Hindu-Recht befaßte. Dieses wurde bei den nicht-islamischen Indern hauptsächlich von den Brahmanen beherrscht und war in Sanskrit niedergeschrieben.

Es war also eine der Aufgaben der Angestellten der Ostindien-Kompanie und der englischen Richter, sich mit den Rechtsverhältnissen der Hindus zu befassen. So wundert es nicht, als erste englische Sanskrit-Philologen gerade "Writer" (Sekretäre) und "Judges" (Richter) kennenzulernen. Beginnen wir mit Charles Wilkins (1750-1833). Er trat 1770 als Writer in den Bengalen-Dienst der Ostindien-Kompanie. Schnell lernte er die in der dortigen Gegend vorkommenden Sprachen. Wollte man die indischen Texte, wie beispielsweise den 1776 von einer Kommission von Brahmanen unter der Leitung von Warren Hastings zusammengestellten Codex eingeborener Gesetze vervielfältigen, so bedurfte es dazu einer Druckerei mit orientalischen Buchstaben. Wilkins konnte schon im Jahre 1778 eine "Printing-press for oriental languages" einrichten. Er hat sie selbst organisiert und betätigte sich darin auch als Setzer und als Metallurgist und Gießer bei der Herstellung der Lettern. Wilkins wurde in der Indologie aber vor allem dadurch bekannt, daß er das aus dem Epos Mahabharata stammende Lehrstück Bhagavadgita übersetzte. Es erschien 1785 in London und ist die erste Übersetzung eines längeren Sanskrit-Textes.

Die Hindu-Gesetze zu erforschen, hatte sich auch ein zweiter Engländer, der juristische Sekretär Henry Thomas Colebrooke (1765-1837), vorgenommen. Er kam 1782 nach Kalkutta; doch er verließ die junge, aufstrebende Stadt bald wieder und ging nach Purneah, weil sich ihm dort mehr Ruhe bot, das Sanskrit zu erlernen. Im Laufe der Jahre veröffentlichte er einige Abhandlungen über Hindu-Gesetze. Colebrooke wurde auch als Samm- 
ler bekannt; so konnte er im Jahre 1819 der East-India-Company in London zahlreiche indische Handschriften, darunter auch einige medizinische, als Geschenk übergeben (42).

Als dritter unter den frühen englischen Sanskrit-Philologen ist WiLliam JoNes (1746 bis 1794) zu nennen, der 1783 als Richter nach Bengalen kam. Er hat sich mit dem SanskritGesetzbuch des Manu beschäftigt und 1796 in Kalkutta das Buch Institutes of Hindu Law veröffentlicht. Schon vorher schuf er die erste Übersetzung des indischen Dramas Schakuntala. Sie erschien 1789 in Kalkutta. Ein Jahr nach seiner Ankunft in dieser Stadt, 1784, wurde dort die Asiatic Society gegründet. Einer ihrer Zweige beschäftigte sich mit den Sanskrit-Werken. William Jones wurde zum Präsidenten dieser für die Erforschung der indischen Welt so wichtigen wissenschaftlichen Gesellschaft gewählt.

In der zweiten Hälfte des 18. Jahrhunderts stieß man beim Sammeln indischer Werke verschiedentlich auch auf medizinische Texte (43). Auf die Texte von Colebrooke wurde bereits hingewiesen. Sie wanderten schließlich fast alle nach Europa in die entsprechenden Fachbibliotheken. Zur Übersetzung eines größeren medizinischen Werkes kam es damals noch nicht. Es wurde aber durch die Übersetzung anderer Texte wichtige philologische Vorarbeit geleistet, welche die später in Indien wie auch in Europa stattfindende Übersetzung der älteren medizinischen Texte erleichterte.

Wenn in dieser Zeit ein längerer medizinischer Text, in dem beispielsweise ausführlich die Symptome beschrieben, Diagnosen gestellt und vorschriften zur Therapie gegeben waren, noch nicht übersetzt wurde, so gab es doch Übersetzungen von Texten, in denen Heilpflanzen aufgezählt werden. Hierin war die Entwicklung in der von Dr. Hermann und van Rheede begonnenen Richtung weitergegangen.

Um 1723 fuhr der Schotte James Fraser nach Surat, um in Indien Handschriften zu sammeln. Unter dem heimgebrachten Material befand sich auch ein pharmazeutisches Lexikon, das im 17. Jahrhundert von dem Hakiem des Kaisers Schah Jehan namens Nureddin Muhammed Abdullah aus Schiras in Persisch verfaßt worden war. Der Titel des Buches hieß Elfaz Edwijeh, was mit «Materia medica» übersetzt wird. Es enthielt in alphabetischer Reihenfolge Arzneien und Spezereien. Aus der gleichen Zeit des Mogulreiches wurden noch andere Handschriften bekannt, die umfangreiche mehrsprachige pharmazeutische Lexika darstellen (44). Ehe es aber zu einer Übersetzung des Fundes von Fraser kam, vergingen mehrere Jahrzehnte. Sie erschien erst 1793, und zwar von dem Orientalisten in der englischen Armee Francis Gladwin. Dieser war seit 1785 Mitglied der Asiatic Society of Bengal und später Professor am College of Fort William. Sein Werk kam in Kalkutta heraus und hatte den Titel Ulfaz Udwijeh or the Materia medica, in the Arabic, Persian and Hindevy Languages, with an 
English Translation. Wir erhalten hier einen vergleichenden Überblick über englische, lateinische, griechische, syrische, arabische, persische und indische Namen ${ }^{10}$ der einzelnen Arzneimittel.

$\mathrm{Zu}$ dieser Zeit haben sich nicht nur die Engländer mit indischen, besonders mit Sanskrit-Texten beschäftigt. Wie wir schon hörten, hat damals auch die katholische Mission an der Bewältigung des Sanskrits gearbeitet. JoHanN Philipp Wesdin, der 15 Jahre in Indien weilte, wurde bereits als Schöpfer der ersten Sanskrit-Grammatik genannt (Abb.4). Er wandte lange Jahre seine Aufmerksamkeit den Sanskrit-Texten zu, sammelte sie in Malabar und studierte auch noch nach seiner Rückkehr aus Indien die indischen

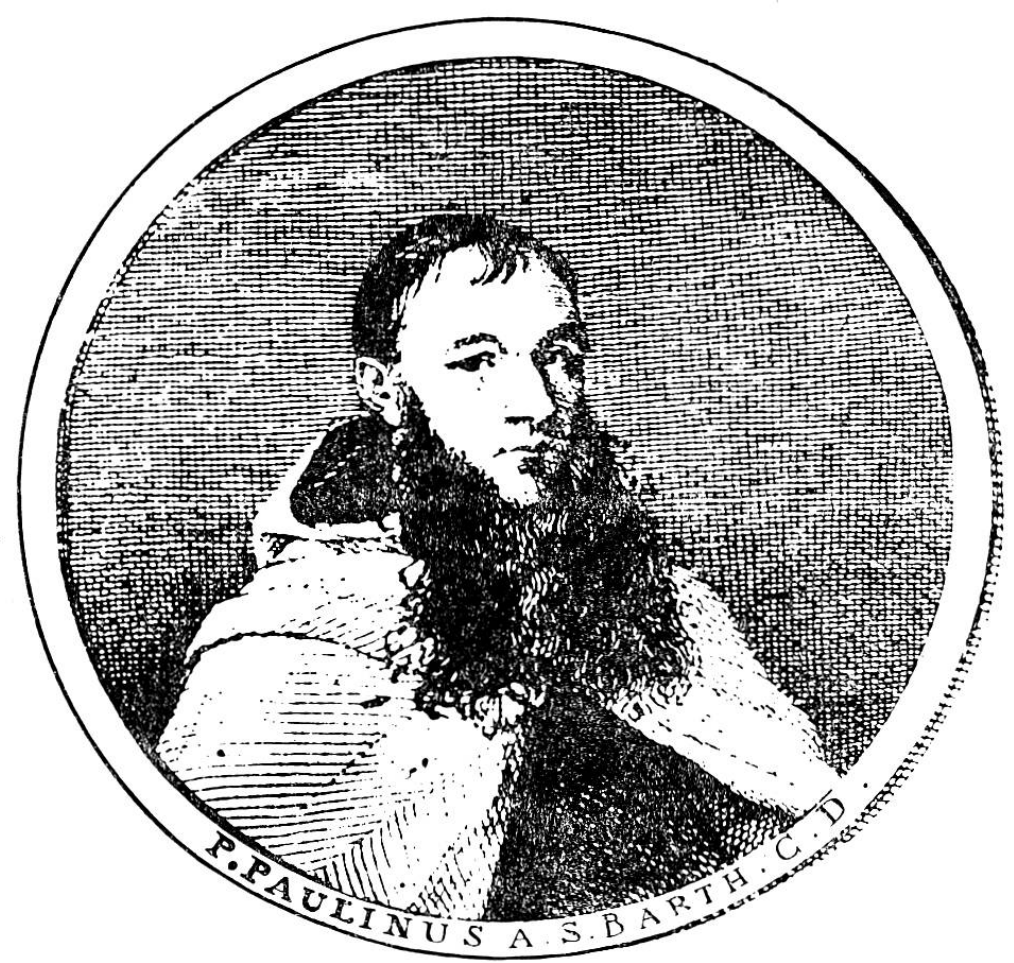

Abb. 4. Johann Philipp Wesdin, der als Karmeliterpater den Namen Paulinus a Sancto Bartholomaeo trug. Er brachte 1790 die erste Sanskrit-Grammatik zu Druck, übersetzte einige medizinische Schlokas und wies auf die riesige Zahl medizinischer Manuskripte in Indien hin (aus Fr. Paolino da S. Bartolomeo, Viaggio alle Indie Orientali, Roma 1796)

10 Nicht nur die Mogul-Kultur hat medizinisch-botanische Wörterbücher geschaffen; es gibt sie auch im Sanskrit. So erwähnten, wie schon berichtet, die drei Brahmanen in van Rheedes Hortus Indicus Malabaricus ihr Wörterbuch Manhaningattnam. Theodor ZACHARIA zählt in seiner 1897 in Straßburg erschienenen Monographie über die indischen Wörterbücher gleich mehrere solcher medizinisch-botanischer Glossare auf, die in Sanskrit geschrieben waren. Vergleiche auch das schon genannte Wörterbuch des Sassetti. 
Manuskripte in der Bibliothek der Congregatio de Propaganda Fide in Rom (dort ist er 1805 gestorben) (45). Für uns ist sein Viaggio alle Indie Orientali, das 1796 in Rom erschien und je eine Übersetzung ins Französische und Englische erfuhr, von besonderem Interesse. Der Reisebericht enthält viele Seiten über Medizin (46) und den aufschlußreichen Satz: «Man findet kein anderes Land auf der Welt, in dem es soviel medizinische Bücher gibt, wie in Indien.»

Die englische Sanskrit-Forschung fand hauptsächlich in Bengalen statt. Wesdin vermittelte in seinem Reisebuch einen Einblick in die Forschung in Malabar. Dort haben sich auch einzelne Portugiesen, möglicherweise Mischlinge und christianisierte, dunkelhäutige Inder, mit Sanskrit-Handschriften beschäftigt. So habe der Pater Joano Alvarez einen SanskritText mit brahmanischer Medizin übersetzt; seine Arbeit sei später von dem Pater Hanxleden fortgeführt worden. Da Hanxleden zwischen 1700 und 1732 in Malabar weilte, war es eine bereits lange vor Wesdin liegende Übersetzung.

In seiner Reisebeschreibung erwähnt Wesdin noch andere Manuskripte. Ihnen allen hat er medizinisch-botanische Namen entnommen. Als Autoren dieser Übersetzungswerke werden der Pater Antonio Gomez, M. Queiros, M. Ambrosio Lopez und ein Vapou genannt. Für die Wiedergabe botanischer Einzelheiten konnte Wesdin Zeichnungen von Pflanzen heranziehen, die von einem malabarischen Arzt angefertigt und von der geistvollen, Botanik liebenden Gräfin voN SAlms mit Anmerkungen versehen waren, sowie ein malabarisches Herbarium, das der Karmeliter-Pater Vincent a Santa Catharina de Siena der Missionsschule des Sankt Pankratius übergeben hatte. Wesdin weist schließlich noch auf einzelne medizinische Schlokas und einige malabarische Palmblattmanuskripte hin, die sich in seinem eigenen Besitz befanden. Drei Sanskrit-Schlokas ${ }^{11}$ werden davon in seinem Reisebuch in lateinischer Transliteration und italienischer Übersetzung gebracht. Sie haben Rezeptcharakter. Zur Zusammenstellung der medizinischen Termini zog er auch «brahmanische Wörterbücher» herbei.

Allein auch Wesdin hat keine Übersetzung eines langen medizinischen Sanskrit-Textes und nicht einmal das Manuskript von Alvarez veröffent-

${ }^{11}$ Schloka heißt eigentlich Spruch, Vers oder Aphorismus. Die Inder haben erst spät die europäische Rezeptform übernommen. Das Rezept nach unserer Art heißt in weiten Teilen Indiens nicht Schloka, sondern «Doktor ka nußcha», was «des Doktors Rezept» heißt. 
licht. In medizinischer Hinsicht blieb er bei der namensmäßigen Erwähnung von Pflanzen und Krankheiten (in Portugiesisch, Sanskrit und Tamil) sowie bei der Übersetzung ganz kurzer Texte stehen.

Wesdin wies schließlich sehr richtig darauf hin, daß die indische Medizin hauptsächlich eine Drogenheilkunde sei. Den indischen Ärzten sei es verwehrt, Anatomie zu studieren. Dafür hätten sie aber in besonderer Weise ihr Augenmerk auf Medikamente gerichtet, und so sei es gekommen, daß sie auf diesem Gebiet mehr verstünden als irgendein Arzt in Europa.

Das Studium der indischen Materia medica erreichte zu Anfang des 19. Jahrhunderts ihren ersten Höhepunkt in dem Werk von Whitelaw Ainslie. Als er 1788 als Garnisonschirurg nach Indien kam, war die Asiatic Society von Kalkutta bereits einige Jahre alt, und Ainslie fand dort schon mehrere Engländer beim Studium indischer Sprachen und Texte vor. Sie konnten ihm also sogleich fachkundige Hilfe leisten. Wie die meisten Leute, die ein Werk über die indische Materia medica schrieben, so hat auch Ainslie zunächst viele Jahre hindurch nur gesammelt. Endlich, 1813, war sein Werk fertiggestellt. Es erschien in Madras unter dem Titel Materia Medica of Hindoostan and Artisan's and Agriculturist's Nomenclature. Dreizehn Jahre später kam es zu einer zweiten, erweiterten Auflage (47). Bis zu Ainslie war bereits viel Material in die Literatur eingegangen. Er sammelte und ergänzte es in mannigfacher Weise. Auch bei ihm finden sich die indischen Namen medizinischer Drogen und Krankheiten ausführlich angeführt. Auf das englische und lateinische Wort folgen die Bezeichnungen in Tamil, Telugu, Dekkani, Mahratti und Sanskrit. Ainslie ging in Basare und besuchte Väidjas. Dort, wie auch aus Sanskrit-Büchern, aus Büchern in der Tamil-Sprache, aus persischen und arabischen Büchern, lernte er immer neue Drogen und deren Anwendung kennen. Unter dem Begriff Buch hat man aber keineswegs ein nach europäischer Art gedrucktes und gebundenes Werk zu verstehen, sondern eine Art Palmblattmanuskript (Abb. 5). Besonders wertvoll bei Ainslie sind die Listen der ihm bekanntgewordenen medizinischen Texte Indiens.

Ainslie hat es verstanden, zahlreiche Leute, darunter einige namhafte Persönlichkeiten, von denen die Orientalisten Charles Wikins und WiLliam Tolfrey genannt seien, zur Mitarbeit zu begeistern. Der zweite wirkte seit 1805 auf der 1795 den Holländern abgenommenen Insel Ceylon, wo er später Chefdolmetscher in der singhalesischen Königsstadt Kandy wurde. Tolfrey stellte für Ainslie eine Liste mit medizinischen Werken zusammen, die sich in den Händen der «Native practitioners» von Ceylon befanden. 


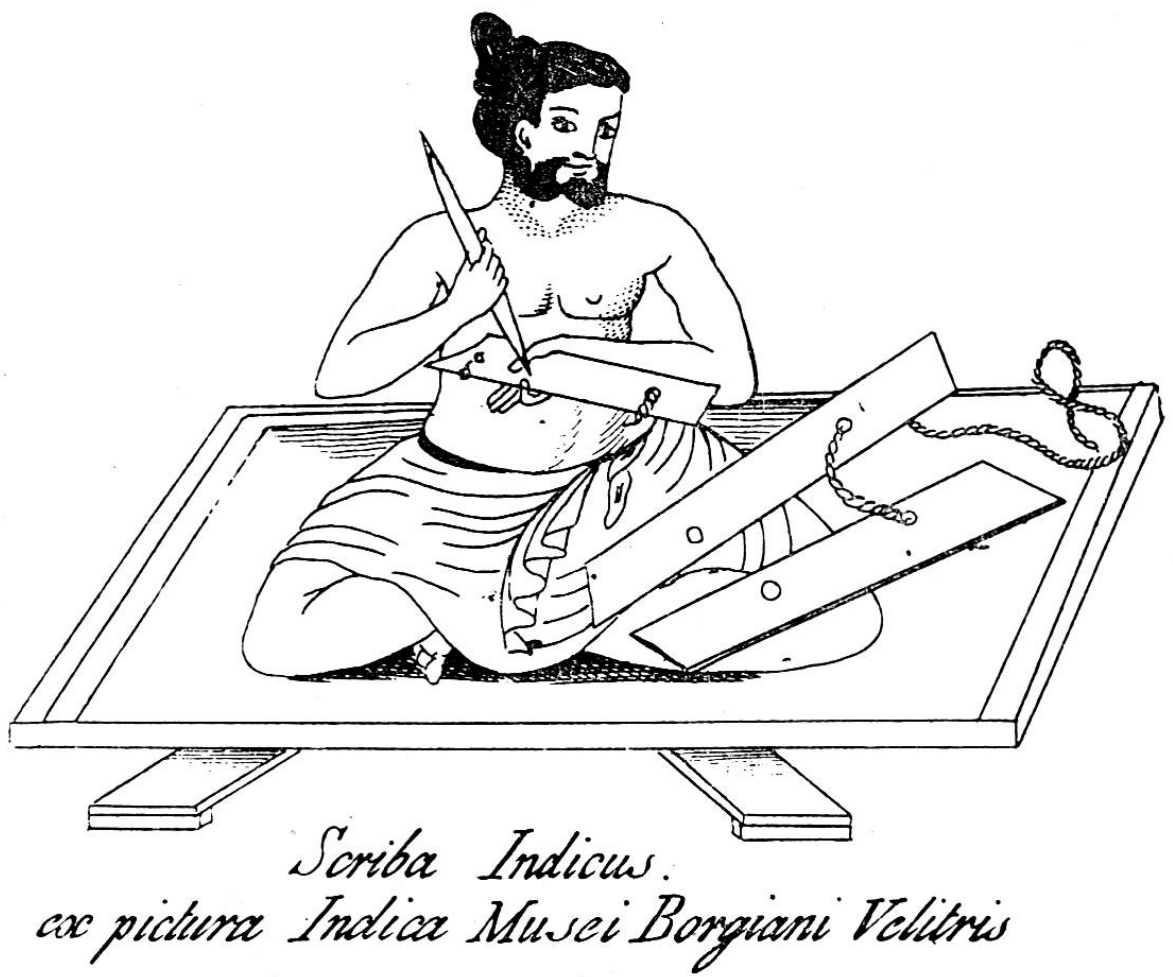

Abb. 5. Schreiber eines indischen Buches. Zwischen zwei steifen Deckeln werden die handgeschriebenen Blätter eingefügt. Das ganze wird durch zwei Schnüre zusammengehalten, die durch ein Loch im Blatt gezogen werden (aus Paolino dA S. BARTolomeo, Viaggio..., 1796, S. 112)

Dabei ergab sich, daß die meisten Texte zwar in Sanskrit abgefaßt, aber in singhalesischen Buchstaben geschrieben waren. Ainslie konnte ferner bereits gedruckt vorliegende Kataloge für seine Literaturliste verwenden, so den 1809 in Cambridge gedruckten Descriptive Catalogue of the Oriental Library of the late Tippoo Sultan of Mysore. Er war von dem Orientalisten Charles Stewart (seit 1781 in der Bengal Army der East India Company) zusammengestellt. Schließlich sei noch ein Väidja erwähnt, von dem Ainslie eine Liste medizinischer Sanskrit-Werke erhielt, und ein Väidja, der eine Zusammenstellung von medizinischen Werken in Tamil beisteuerte. Was nützten aber bei der Abfassung der Materia medica all die Vokabeln, Listen und Bibliothekskataloge? Sie sind nur Hilfsmittel. Im Vordergrund steht die Droge selbst, die bei der indischen Materia medica meist eine Pflanze war. Sie mußte botanisch und pharmakologisch eingeordnet werden. Bei dieser Arbeit stand Ainslie ein deutscher Botaniker, Doktor J.P. RotTlen, helfend zur Seite. Ihm widmete Ainslie dann auch sein Werk. 


\section{Zusammenfassung}

Über dreihundert Jahre hat es gedauert, bis das erste große indische medizinische Werk vollständig zum Druck kam. Es war Suschruta. Durch seine Vervielfältigung war es nun in Indien wie in Europa möglich, Übersetzungen vorzunehmen und diese philologisch auf ihre Richtigkeit hin miteinander zu vergleichen. Den Anfang machte der bayrische Amtsarzt Dr. Franz Hessler (1799-1890) (42). Mit großem Fleiß und viel Mut übersetzte er nach und nach große Teile des Suschruta ins Lateinische; 1844 erschien der erste Band seines Buches, 1847 der zweite - beide in Erlangen. Heßler fand viel Kritik, vernichtende Kritik! Ist das zweibändige Buch in philologischer Hinsicht ein großer Mißerfolg gewesen, so gebührt ihm doch das Lob, daß es der philologischen Detailarbeit das Tor zu einem weiten Feld aufgestoßen hat. Nach und nach wurden die Fehler aufgeklärt und die Sätze philologisch präzisiert. Es kamen englische und deutsche Übersetzungen heraus. Einzelne Kapitel wurden nach fachärztlichen oder andern Gesichtspunkten bearbeitet. Als Resultat liegt heute eine riesige Literatur zum Suschruta vor.

Die erste Druçklegung des Suschruta erfolgte durch einen Inder und nicht durch einen Europäer. Dies steht in bemerkenswertem Gegensatz zu dem großen Interesse, das die Europäer an der indischen Medizin über dreihundert Jahre hinweg gezeigt haben; hatten sie doch nicht zuletzt wegen der berühmten indischen Heilkunde, wegen der medizinischen Drogen die strapaziösen Seefahrten nach dem fernen Indien unternommen. Als MadhusuDANA Gupta 1835 den ersten Band des Suschruta herausbrachte, waren die wesentlichen Einzelheiten der indischen Materia medica sowie der ärztlichen Behandlungsmethoden (z.B. durch Diät und Starstich) in der europäischen Literatur bereits längst wissenschaftlich erfaßt. Es erschien aber reizvoll, in diesem Aufsatz einmal zu zeigen, wie sich die Erforschung dieser Dinge sprachlich vollzogen hat. Wie schon zu Anfang festgestellt wurde, fand sie nicht in der stillen Stube durch philologisch detailliertes Studium von Texten statt, sondern draußen in der Praxis, am Patienten oder an Drogen. In Indien beheimatete Ärzte, Apotheker oder Dolmetscher gaben dem fragenden Europäer die sprachliche Erklärung, nannten die einheimischen Namen für die Pflanzen und Krankheitserscheinungen und beschrieben dann in portugiesischer, holländischer oder englischer Sprache weitere Einzelheiten wie beispielsweise die Zubereitung und Anwendung der einzelnen Medizinen. 
Wie er nach indischer Medizin fragte, davon hat GARCIA DA ORTA eine schöne Schilderung gegeben. Indem er sein Buch über die indische Medizin in die Form eines Kolloquiums brachte, war es möglich, beispielsweise einen Jungen aus dem Volk, eine Dienerin im portugiesischen Haushalt, einen Patienten und einen Arzt in der ihnen eigenen Weise sprechen zu lassen. Das Werk Ortas beschränkte sich also nicht auf eine sachliche Aufzählung medizinisch-pharmakologischer Daten, sondern schildert in ungezwungen lebendiger Weise auch das Milieu, in dem solche Daten in Erfahrung zu bringen waren ${ }^{12}$.

Während uns bei Garcia da Orta einige seiner Gewährsleute dergestalt vorgeführt werden, daß ihre direkte Rede wiedergegeben wird, lernen wir die Gewährsleute von Hendrik van Rheede in seinem Hortus Indicus Malabaricus auf andere Weise kennen. Sie stellen sich selbst vor, und zwar indem sie in ihrer eigenen Sprache und Schrift einen Bericht über Namen, Beruf und Herkunft abgeben. Sie sagen schließlich auch, in welcher Weise sie van Rheede bei der Erarbeitung des Materials geholfen haben.

Insgesamt läßt sich aus den bis zum Anfang des 19. Jahrhunderts gedruckten Werken über die Geschichte der Erforschung der indischen Medizin folgendes sagen: Zunächst beschränkten sich die Autoren auf die Erforschung der indischen Namen, beispielsweise der Heilpflanzen. Wußte man den Namen und sagte ihn einem Inder in mehr oder weniger gutem Indisch, so erhielt man von ihm die gewünschte Pflanze. Dies reichte für den Handel aus. Spätere Forscher legten dann Wert darauf, daß die Namen der Pflanzen auch in der einheimischen Schrift aufgeschrieben wurden. So

12 Wir finden in der zweiten Hälfte des 16. Jahrhunderts mehrere europäische medizinische Bücher in der Form eines Dialogs oder Kolloquiums. Man denke an das 1591 erschienene Werk des Arztes und Ägyptenreisenden Prosper Alpinus (1533-1616) De Medicina Aegyptorum. Es wäre vielleicht interessant, einmal nachzusehen, wann in Europa mit dieser Art des Abfassens von wissenschaftlichen Texten begonnen wurde. Bei Garcia da Orta läßt sich indischer Einfluß vermuten. Die indischen Ärzte faßten ihre medizinischen Texte gern in dichterischer Weise, sogar in Versen ab; dabei ließen sie verschiedene Leute berichten. Dem portugiesischen Arzt mag das imponiert haben. Er hat bei den Indern wohl auch erfahren, daß sie den als besonders guten Arzt ansahen, der ein medizinisches Thema nicht nur sachlich, sondern auch in dichterischer Weise niederschreiben konnte. Ein solcher Arzt heißt Kaviradsch. Kavi heißt dabei Dichter und Radsch der König, Kaviradsch also Dichterkönig. Dieses Wort hat im Laufe der Jahre einen Begriffswandel durchgemacht; der ursprüngliche Sinn ist abgeblaßt. Frägt man heute in Indien jemand nach einem Kaviradsch, so wird man auf einen wohlerfahrenen, praktischen Arzt verwiesen - und dieser schreibt meist überhaupt keine Bücher mehr. 
finden wir bei van Rheede die Sanskrit-Namen in Sanskrit, die arabischen Namen in Arabisch und die Tamil-Namen in Tamil neben die Abbildungen gesetzt.

Lange Zeit beschränkte man sich auf das Sammeln der Namen. Indische Wörterbücher standen hier helfend zur Seite. Erst in der dritten Phase begann man damit, auch indisch-medizinische Texte zu übersetzen. Hier seien Joano Alvarez und Johannes Gründler genannt. Das Manuskript des ersten kam nicht zum Druck. Die von Gründler unternommene Übersetzung ausgewählter Kapitel erfuhr nur in ganz kleinen Bruchstücken eine Drucklegung. Sie stellt eigentlich nicht einmal eine U̇bersetzung dar, weil nur Auszüge aus den Übersetzungen stichprobenartig wiedergegeben werden. Gründler konnte auf diese Weise größeren philologischen Schwierigkeiten aus dem Wege gehen. Sein Werk muß darum mit sehr kritischen Augen betrachtet werden. Gründler selbst beließ es bei dieser einen Kompilation. Als ihn der Hallenser Orientalist Christian Benedikt Michaelis später um weitere medizinische Übersetzungen bat, antwortete Gründler am 8. September 1717 - in Anbetracht der philologischen Schwierigkeiten einer neuerlichen Übersetzung ausweichend-, daß er keine Zeit dafür hätte (48). Immerhin dürfen wir sagen, daß die erste durch Druck veröffentlichte Übersetzung ausgewählter Kapitel indischer Texte 1712 durch Gründler erfolgte. Einen Einfluß auf die medizinische Praxis der deutschen Ärzte hat die Übersetzung Gründlers allerdings nicht gehabt.

Als endlich nach 1835 die philologisch-detaillierte Bearbeitung des Suschruta und später noch anderer Texte begann, war ärztlicherseits das praktische Interesse daran so gut wie ganz erloschen. Das Echo blieb aus. Die Zeit, in der die europäischen Ärzte und Apotheker etwas Neues von der indischen Heilkunde für ihre Therapie erfahren wollten, war vorbei. Man hatte sich in den vergangenen dreihundert Jahren ohne genaue Kenntnis der grammatikalischen und philologischen Details behelfen müssen ${ }^{13}$. Die

${ }^{13}$ Als 1823 in Kalkutta die "Medical and Physical Society" zur Erforschung medizinischer Dinge in Indien gegründet wurde, hatte man einen Aufruf erlassen. Darin wurde um Mitarbeit zur Erfassung der medizinischen Topographie und der Meteorologie, der Krankheiten, der einheimischen chirurgischen Instrumente, der Materia medica usw. gebeten. Man erinnerte auch daran, über noch unbekannte Behandlungsmethoden der Native practitioners zu berichten (The Oriental Magazine and Calcutta Review 1 [1823] 617-9, Trans. Med. Physic. Soc. Calcutta, in deren erstem Band 1825).

Im Hinblick auf die indische Medizin dachte man in der Society vornehmlich an eine praktische Erforschung in der Art, wie sie bisher betrieben war. Die Society wies nämlich 
hauptsächlichsten Dinge waren schon durch einfaches Befragen indischer Ärzte und Apotheker und durch Beobachtung der Behandlung ihrer Patienten in Erfahrung gebracht worden. So ist die Erforschung der indischen Medizin an den großen Werken der indischen medizinischen Literatur vorbeigegangen. Das Studium der Texte ist seit 1835 nur noch eine Sache der philologisch arbeitenden Indologen und Medizinhistoriker gewesen. Man maß ihm in Europa kaum noch eine direkte Bedeutung für den Fortschritt der ärztlichen Praxis zu. Und weil bei diesem Studium der historische Akzent überwiegt, wandten die Indologen und Medizinhistoriker, wie schon erwähnt, nur den großen Texten der alten klassischen Zeit der SanskritMedizin ihre Aufmerksamkeit zu. Die vielen medizinischen Texte, die in Indien in den letzten zweihundertfünfzig Jahren und schon früher abgefaßt wurden, liegen nach wie vor unbeachtet in den Bibliotheken oder gingen in den vergangenen Jahrzehnten zum großen Teil verloren.

Die Inder selbst hielten in den letzten hundert Jahren den Inhalt ihrer jüngeren medizinischen Schriften im Vergleich zu den klassischen Werken und der importierten europäischen Fachliteratur für unwichtig und versprachen sich von ihnen keinen Vorteil mehr. Das lag an der Zeit. Viele junge Inder sahen damals das Sanskrit-Studium als rückständig und darum als Hemmnis an. Das Studium der westlichen Medizin war Trumpf und Englisch die Sprache des Fortschritts. Die medizinischen Texte, die nicht Sanskrit waren oder aber bei denen das Sanskrit mit südlichen Schriftzeichen geschrieben war, fanden auch kaum noch das Interesse der europäischen Indologen. Das hatte allein schon sprachliche Gründe; denn unsere Indologen können meist nur Sanskrit und Pali, bestenfalls noch Bengali oder Hindi. Da sie mit den andern Texten sprachlich nicht recht umzugehen vermögen, waren sie uninteressant. Die südlichen, meist handgeschriebenen Texte wurden deshalb nur selten von Indologen angekauft und kamen daher auch nicht mehr in den Manuskriptenhandel. Sobald sie unansehnlich wurden, warf ihr Besitzer sie fort.

Zum Schluß sei auf die in Südindien auch heute noch erfolgende Herstellung medizinischer Literatur aufmerksam gemacht. Während meiner Reise 1961 konnte ich allein schon in der Provinz Kanara (Mysore State) mehrere erst neuerdings gedruckte medizinische Texte in kanaretischer

nicht besonders auf die Übersetzung von indischen Texten hin. Sie waren das Objekt der mehr philologisch ausgerichteten Asiatic Society, mit deren Unterstützung es schließlich in Kalkutta zur Herausgabe des Suschruta gekommen ist. 
Sprache feststellen. Sie enthielten eine Fülle an pharmazeutischen Dingen, auch Präparate, die in einheimischen Fabriken hergestellt werden. In Karwar übersetzte mir der Väidja und Drogenhändler Damodar NAGAPPA Schanbay Einzelheiten aus derartigen Büchern. Sie erinnerten zwar an das, was man bei uns Naturheilverfahren zu nennen pflegt, und an den Heilpraktiker, $\mathrm{ja}$ in mancher Weise auch an die Homöopathie, überraschten mich in ihrer Art aber doch und scheinen eines genaueren Studiums wert zu sein. Sie legen Zeugnis davon ab, daß es in Indien noch immer Leute gibt, die ein eigenes medizinisches System befürworten und versuchen, dies auch wissenschaftlich zu erweitern.

\section{Schrifttumsverzeichnis}

(1) J.B. Amancio Gracias, Medicine in Goa in XVI.-XVIII. centuries, Arquivos da Escola Medico-Cirurgica de Nova Goa, Ser. A, 1941, Fasc. 15, S. 234.

(2) Edgar Thurston, Castes and Tribes of Southern India, Band 1, Madras 1909, S. 341.

(3) Heinz Bechert, Sanskritliteratur bei den Singhalesen, Verhandlungen der Indologischen Arbeitstagung in Essen-Bredeney 1959, Göttingen, S. $225 \mathrm{ff}$.

(4) Jogendra Nath Bhattachandra, Hindu Castes and Sects, Calcutta 1896, S. 158-71.

(5) Antonio da Silva Rego, Documentaçao para a Historia das Minoes do Padroado do Oriente India, Band 1, Lisboa 1947, S. 95, 300-6, $358 \mathrm{f}$. und $365 \mathrm{ff}$.

Pedro José da Silva, Elogio historico e noticia completa de Tomé Pires, pharmaceutico e primeiro naturalista da India, Lisboa 1866.

Armando Cortesao, The "Suma oriental" of Tomé Pires, Hakluyt Society, London 1944, 2 Bände; Primeira Embaixada Europeia a China. O Boticario e Embaixador Tomé Pires..., Lisboa 1945.

Condo Don Francisco de San Luiz, J. Soc. Pharm. Lusit. 2 (1838) 36.

Friedrich Flǘckiger und Daniel Hanbury, Pharmacographia, 2. ed., London 1879, S. 681 .

Friedrich Flǘckiger, Indische Pharmakognosie, Arch. Pharm. 22 (1884) $24.9 \mathrm{ff}$.

(6) Alberto Carlos Germano da Silva Correia, Le Cholera aux Indes Portugaises, Arquivos da Escola Medico-Cirurgica de Nova Goa, Ser. A, 1929, Fasc. 4, S. $425 \mathrm{ff}$.

F. W. Rieppel, Zur Frühegeschichte der Rauwolfia, Sudhoffs Arch. Gesch. Med. Naturwiss. 40 (1956) $231 \mathrm{ff}$.

Hans Schadewaldt, Zur Geschichte der Rauwolfia, Veröff. Intern. Ges. Gesch. Pharm. 13 (1958) $139 \mathrm{ff}$.

(7) Garcia da Orta, Coloquios dos Simples e Drogas da India. Die Ausgabe ist dirigiert und mit Anmerkungen versehen worden von Conde de FicalHo, Lisboa 1891, S. 261.

(8) Ebenda, Col. 54, 328-32.

(9) Johannes Boecler, Pauli Hermanni Cynosura Materiae Medicae, Argentorati 1726. J.F. Royle, Ein Versuch über das Altertum der indischen Medizin (übersetzt von J. Wallach), Cassel 1839, S. 9. 
(10) H.A. Hoppe und W. Herrmann, Tropische Gewürzdrogen, München 1949, S. 29-34.

(11) Theophilo Braga, Historia da Universidade de Coimbra, Band 1, Lisboa 1892, S. 434.

(12) J.B. Amancio Gracias, Médicos europeus em Goa e nas cortes indianas nos séculos XVI a XVIII. Separata d'Oriente Portuguez, Bastora 1939.

(13) J. Gerson da Cunha, The Origin of Bombay, Bombay 1900, S. 100.

(14) Angelo de Gubernatis, Storia dei viaggiatori italiani nelle Indie orientali, Livorno 1875; Matériaux pour servir à l'histoire des études orientales en Italie, Paris/Rom 1876.

(15) Jасов Bontius, De medicina Indorum, Leyden 1642, Paris 1646; Historiae naturalis et medicae Indicae Orientalis, Amstelodami 1658.

(16) Christoph Schweitzer, Journal- und Tagebuch seiner sechsjährigen Ostindianischen Reise, Tübingen 1688, S. 121.

(17) S.J. van Oostroom, Hermann's Collection of Ceylon Plants in the Rijksherbarium (Natural Herbarium) at Leyden, Blumea, Suppl. I, Leiden 1937, Sonderdruck.

(18) Paul Hermann, Catalogus Horti Academici Lugduno-Batavi ... Descriptiones et Icones, Leiden 1687.

L. Zumag, Florae Lugduni-Batavae flores, Leyden 1690.

Paul Hermann, Paradisus Batavus, Leiden 1698; Musaeum Zeylanicum, sive catalogus plantarum in Zeylana sponte nascentium, Lugduni Batavorum 1717.

J. Burman, Thesaurus Zeylanicus, Amsterdam 1737.

Carolus Linnaeus, Flora Zeylanica, sistens plantas indicas Zeylone insulae; quae olim 1670-1677 lectae fuere a Paulo Hermanno ..., Holmiae 1747.

P.C. Molhuysen, Bronnen tot de Geschiedenis der Leidsche Universiteit, 4./5.Teil, S'Gravenhage/Leiden 1921, S. 87.

(19) Anant Kakba Priolkar, The Printing Press in India, its Beginnings and Early Development, Bombay 1958.

(20) Zur Geschichte derSanskrit-Philologie siehe: Ernst WIndisch in: Grundriß der IndoArischen Philologie und Altertumskunde, 1. Band, 1. Heft, Straßburg 1917.

(21) Dazu: Franz Caspar Schillinger, Persianische und Ostindianische Reis, Nürnberg 1707.

(22) wie (20).

(23) Wie (19), S. 32.

(24) Lit. deutsche Übersetzung, S. 186.

(25) Vgl. hierzu: Großes vollständiges universales Lexikon, Band 19, Halle/Leipzig 1739.

(26) D. Arno Lehmann, Alte Briefe aus Indien, Berlin 1957, S. $112 \mathrm{f}$.

(27) Ebenda, S. 224.

(28) Ebenda, S. 269.

(29) Ebenda, S. 249.

(30) Ebenda, S. 289.

(31) Lit. z. B. Paulino da SAN Bartolomeo, Reise nach Ostindien (aus dem Italienischen); in: Magazin von merkwürdigen neuen Reisebeschreibungen (Berlin) 15 (1798) 77 und viele andere Autoren. 
(32) J.N. Gloyer, Fragmente über Ostindien, Altona 1813, S. 245.

(33) James H. Gense, A Cocise History of India, Bombay/.../London 1961, S. 313.

(34) Seyed Nurullah und J.P. NaIK, A History of Education in India (during the British Period), 2. ed., Bombay/.../London 1951, S. 92.

(35) Goodeve Chuckerbutty, The Present State of the Medical Profession in Bengal, Brit. Med. J. 2 (1864) 88.

(36) A.F. Rudolf Hoennle, The Bower Manuscript, Facsimile leaves, Nagari Transcript, Kalkutta 1893-1912.

(37) M. Winternitz, Geschichte der indischen Literatur, 3. Band, Leipzig 1920, S. 552. Dazu: Ruston Jal Vakil, Early Contributions to the Study of the Pulse, Indian J. Hist. Med. 1 (1956) 22-8, und andere Autoren.

(38) Narendra Nath Law, Promotion of Learning in India during Muhammedan Rule, London 1916, S. 77.

D. V. Subba Reddy, Influence of Indian Medicine and Arabian and Persian Medical Literature, Indian J. Hist. Med. 4 (1959) 31.

(39) Hakim Abdul Wahab Zahuri, Medical Aid, Medical Men and Medical Writings of the Qutub Shahi Period, Indian J. Hist. Med. 5 (1960) 43-9. A Rare Medical Manuscript of 16th Century A. D., Indian J. Hist. Med. 4 (1959) 25-8.

D. V. Subba Reddy, Dar-us-Shiva (House of Cures), Indian J. Hist. Med. 4 (1959) 103.

(40) François Bernier, Voyages... contenant la description des états du Grand-Mogol, de l'Indoustan, du royaume de Cachemire, Amsterdam 1699, 1710, 1724; Travels in the Mogul Empire (übersetzt von A. Constable) Westminster 1891.

(41) Anquetil du Perron, Abraham Hyacinthe: Reisen nach Ostindien nebst einer Beschreibung der bürgerlichen und Religionsgebräuche der Parsen (deutsch von PurmanN), Frankfurt 1776.

(42) Vgl. Franz Hessler, De antiquorum Hindorum medicina et scientiis physicis, quae in Sanscritis operibus exstant, Würzburg 1830.

(43) William Jones, Abhandlungen über die Geschichte und Altertümer, die Künste, Wissenschaften und Literatur Asiens (deutsch von J. CHr. FICK), 1. Band, Riga 1795, S. 279.

(44) The Asiatic Journal and Monthly Register, Band 1 der neuen Serie, London 1830. Cyril Elgood, A Medical History of Persia and the Eastern Caliphate, Cambridge 1951.

(45) Paulinus a Sancto Bartholomaeo, Examen historico-criticum Codicum Indicorum Bibliothecae Congregationis de Propaganda Fide, Roma 1792.

(46) Paulin de S. Barthélmy, Voyage aux Indes Orientales, 2. Band, Paris 1808, S. 419-76.

(47) Whitelaw Ainslie, Materia Indica; or, some account of those articles which are employed by the Hindoos, and other Eastern Nations, in their medicine, arts and agriculture; comprising also formulae, with practical observations, names of diseases in various eastern languages, and a copious List of Oriental Books immediately connected with general science, etc., Band 1 und 2, London 1826.

(48) Wie (26), S. 489. 